CERN-TH/96-158

UTHEP-96-0601

\title{
Upgrade of the Monte Carlo program BHLUMI for Bhabha scattering at low angles to version $4.04^{\S}$
}

\author{
S. Jadach \\ Institute of Nuclear Physics, ul. Kawiory 26a, Kraków, Poland \\ CERN, Theory Division, CH-1211 Geneva 23, Switzerland \\ W. Płaczek ${ }^{\dagger}$ \\ Department of Physics and Astronomy, \\ The University of Tennessee, Knoxville, TN 37996-1200, \\ E. Richter-Wąs \\ Institute of Computer Science, Jagellonian University, \\ Kraków, ul. Reymonta 4, Poland, \\ CERN, Theory Division, CH-1211 Geneva 23, Switzerland \\ B.F.L. Ward \\ Department of Physics and Astronomy, \\ The University of Tennessee, Knoxville, TN 37996-1200, \\ SLAC, Stanford University, Stanford, CA 94309

\section{Z. Wạs} \\ Institute of Nuclear Physics, Kraków, ul. Kawiory 26a, Poland, \\ CERN, Theory Division, CH-1211 Geneva 23, Switzerland
}

\begin{abstract}
The new version of the Monte Carlo program for small-angle Bhabha scattering BHLUMI with an overall precision of $0.11 \%$ is presented. The main improvements are in the matrix element of the main multi-photon sub-generator based on the Yennie-Frautschi-Suura exponentiation, where the missing second-order terms in the leading-logarithmic (LL) approximation are supplemented and the contribution from the $Z$ resonance is improved. In the LUMLOG sub-generator based on the LL approximation, we implement collinear photon emission in the final state and we also add a down-graded third-order LL matrix element, which has exactly the same incomplete third-order LL contribution as the multi-photon BHLUMI. The new LUMLOG is therefore capable of calculating the missing third-order LL part in the cross sections obtained from the main multi-photon event generator, for arbitrary event selection. The first-order event generator OLDBIS remains unchanged.
\end{abstract}

\section{Submitted to Computer Physics Communications}

$\S$ Work supported in part by the US DoE contract DE-FG05-91ER40627, Polish Government grants KBN 2P30225206, 2P03B17210, the European Union contract No. ERB-CIPD-CT94-0016 and Polish-French Collaboration within IN2P3.

$\dagger$ On leave of absence from Institute of Computer Science, Jagellonian University, Kraków, Poland.

\section{CERN-TH/96-158}

UTHEP-96-0601

June 1996 


\section{NEW VERSION SUMMARY}

Title of the program: BHLUMI version 4.04

Reference to original program: Comput. Phys. Commun. 70 (1992) 305

Computer: IBM rs6000, HP9000 and other UNIX workstations

Operating system: UNIX

Programming language used: FORTRAN 77

High speed storage required: $<5 \mathrm{MB}$

No. of bits in a word: 32

Peripherals used: Line printer

No. of cards in combined program and test deck: about 12000

Keywords: Radiative corrections, Monte Carlo simulation, Bhabha scattering, bremsstrahlung, Quantum Electrodynamics (QED), electroweak theory, structure functions.

Nature of physical problem: The small-angle Bhabha scattering process is used in all electron-positron colliders to calculate machine luminosity. This process is subject to QED radiative corrections, which have to be known for arbitrary cut-offs and/or acceptance with a precision at least a factor of 2 better than the pure experimental precision. This means that the level of $0.05 \%$ should be reached. A realistic simulation should include multiple emission of the bremsstrahlung photons.

Method of solution: The Monte Carlo simulation of the small-angle process is an ideal solution. It provides the integrated cross-section for arbitrary cuts. Direct simulation of the final state electrons and photons is precisely what is needed for detector simulation purposes.

Restrictions on the complexity of the problem: The overall precision of the QED calculation is restricted to $0.11 \%$, for a typical LEP/SLC luminometer angular range of $1^{\circ}<\vartheta<3^{\circ}$, where $\vartheta$ is the scattering angle.

Typical running time: The efficiency for multi-photon sub-generator is typically 6 million variable-weight events and 4 million constant-weight events per hour for an HP9000$735 / 100 \mathrm{MHz}$ machine. 


\section{LONG WRITE-UP}

\section{Introduction}

At high energy electron-positron scattering experiments such as those now taking data at LEP and the SLC and those planned for the future NLC, in order to translate the number of events into a cross-section one has to know the luminosity of the accelerator at the interaction point. The luminosity is deduced from a measurement of the number of events for one (or more) scattering process(es) for which the integrated cross-section is safely calculable from the theory. The $e^{+} e^{-}$elastic scattering, the so-called Bhabha process, is a very good candidate because in the range of a few degrees it is dominated by perfectly known physics, the exchange of a $t$-channel photon. It is therefore routinely used in all $e^{ \pm}$colliders for luminosity measurement. Note that, in order to keep statistical errors small, the process used for luminosity measurement has to have a large enough cross-section. This condition is also fulfilled in the Bhabha small-angle scattering process rather easily, by putting the detector at small enough angles.

The small-angle Bhabha process ( $\mathrm{SABH}$ ), as any high energy process with light charged fermions, is subject to relatively large, a few per cent, QED radiative corrections. In the experimental luminosity measurement, these corrections have to be calculated and their effect taken into account, in other words removed from the luminosity experimental value. These effects have to be calculated with a precision comparable to or better than the best experimental error, which for single LEP experiments is about $0.1 \%$ and for combined results of the four LEP collaborations is at the $0.05 \%$ level [1]. The new version of the Monte Carlo event generator BHLUMI presented here marks a definite step forward in the direction of sub-per mille theoretical error for the SABH process.

As compared to the previous version, 2.02, presented in Ref. [2], this new version features several important improvements, which will be described in detail. Improvements are mainly in the matrix element, while in the basic (low-level) Monte Carlo (MC) algorithm there are only minor improvements, which will be explained in detail. The general description of the basic MC algorithm will not be repeated and the reader should look into Ref. [2] for more details on technicalities of the low-level MC algorithm.

The main improvements in the program are:

- Inclusion of the missing second-order leading-logarithmic (LL) correction, $\mathcal{O}\left(\gamma^{2}\right)$, where $\gamma=2 \frac{\alpha}{\pi}\left[\ln \left(|t| / m_{e}^{2}\right)-1\right]$, in the exponentiated (multi-photon) matrix element according to Ref. [3].

- Implementation of the matrix element without exponentiation (for tests), which is defined in Ref. [3] and corresponds strictly ${ }^{1}$ to the matrix element with exponentiation.

- Improvement of the QED corrections to the $Z$ contribution according to Ref. [4].

\footnotetext{
${ }^{1}$ The same $\mathcal{O}(\alpha \gamma)$ cotributions.
} 
- Implementation of the final state collinear radiation with the matrix element up to $\mathcal{O}\left(\gamma^{3}\right)$ with and without exponentiation in the LUMLOG sub-generator. LUMLOG generates photons that are strictly collinear to the parent initial or final state electron (positron).

- Inclusion in the LUMLOG sub-generator of the truncated $\mathcal{O}\left(\gamma^{3}\right)$ matrix element, which corresponds to the exponentiated multi-photon matrix element of BHLUMI (it is obtained by means of exact analytical integration over the transverse photon momenta), see also Ref. [5].

- Inclusion of the best calculation of the vacuum polarization according to Refs. [6-8].

Let us add the following important remarks: Inclusion of the missing $\mathcal{O}\left(\gamma^{2}\right)$, secondorder LL correction was done very carefully, with many cross-checks, which are presented in Refs. [3,5], see also Ref. [9]. They are based on comparisons of the Monte Carlo results with semi-analytical calculations; in the full form they will be published in Ref. [10]. According to the conclusions of the Bhabha Working Group of the 1995 LEP2 Workshop (see Ref. [11] and its summary published in Ref. [12]) the present program BHLUMI 4.04 features a precision of $0.11 \%$ for the best LEP luminometers at the scattering angles $1^{\circ}<\vartheta<3^{\circ}$ at LEP1, and $0.25 \%$ for any kind of typical luminometers in the angular ranges within $1^{\circ}<\vartheta<6^{\circ}$ at LEP2. This estimate is based on comparisons with the other MC calculations, in particular with the SABSPV calculation [13]. As is shown later in this paper, the SABSPV recipe is also implemented (emulated) in the framework of the present BHLUMI package ${ }^{2}$.

In the future version of BHLUMI, see discussions in Ref. [11], it will be necessary to add the missing second-order sub-leading contributions $\mathcal{O}(\alpha \gamma)$, unless they prove to be too small to warrant such attention. The first exercises in this direction were already done, see Refs. [14-16], and we are planning to implement the contributions of $\mathcal{O}(\alpha \gamma)$ in the next version of the program.

The outline of the paper is the following: we describe in detail all the new features of LUMLOG in Section 2, and we give an exact description of all input data and output results in Section 3. In Section 4 we describe the distribution directory with many useful demonstration user programs, which should help the user to learn how to use BHLUMI effectively and easily. Short conclusions end the paper.

As already mentioned, we do not show explicitly in the present paper the new matrix element, because it is already published in Ref. [3] (see also the erratum to that paper).

\section{New LUMLOG}

In the LUMLOG MC event sub-generator, photons are generated with zero transverse momentum (strict collinear approximation) according to LL structure functions. LUMLOG was always used in order to calculate the higher-order corrections missing in the

\footnotetext{
${ }^{2}$ The SABSPV emulation is not part of the BHLUMI MC event generator. It is implemented, using OLDBIS and LUMLOG, in one of the examples of the user programs, see Section 4.
} 
multi-photon sub-generator of the BHLUMI package. In Refs. $[17,18]$ it was used in order to estimate the missing $\mathcal{O}\left(\gamma^{2}\right)$, second-order LL contribution. It was also instrumental in the construction of the so-called OLDBIS + LUMLOG $_{\text {h.o. }}$ cross section, which was compared with the results of multi-photon BHLUMI, see Ref. [18]. The earlier version of the present LUMLOG was already used in Refs. $[11,12]$ in order to determine the missing $\mathcal{O}\left(\gamma^{3}\right)$ leading-logarithmic contribution in the present version of the multi-photon BHLUMI. The actual version of LUMLOG was employed in the recent work of Ref. [5], see also the comment at the end of this section.

The present new version of LUMLOG is changed substantially with respect to the older version of Ref. [2]. The final state bremsstrahlung is now implemented. This requires modification of the generation algorithm. The final state bremsstrahlung is important for all kinds of tests, in particular for so-called non-calorimetric event selections, see [19]. These non-calorimetric event selections are unrealistic, but useful for tests of the matrix element and for comparisons with the other calculations. Another important new feature is the "OO $\left(\gamma^{3}\right)$ emulation" in LUMLOG of the multi-photon matrix element of the multi-photon BHLUMI. What is this "emulation", and why is it useful? To begin an explanation, let us stress that it is feasible to add the missing corrections of $\mathcal{O}\left(\gamma^{3}\right)$ to the matrix element in the multi-photon BHLUMI. The question is whether it is really worth while. We expect that $\mathcal{O}\left(\gamma^{3}\right)$ is almost complete in the cross section from multi-photon BHLUMI, because of the Yennie-Frautschi-Suura exponentiation - this is true for instance for the initial state bremsstrahlung, see Ref. [20]. If, for instance, the missing $\mathcal{O}\left(\gamma^{3}\right)$ in BHLUMI is $10^{-4}$ then it is not worth while to add it explicitly in the matrix element. But how does one know how big the missing $\mathcal{O}\left(\gamma^{3}\right)$ is in BHLUMI, without actually adding it to its matrix element in the first place? The solution is the "emulation" of multi-photon BHLUMI within LUMLOG. First of all, one has to add the complete $\mathcal{O}\left(\gamma^{3}\right)$ in the final state, in addition to the initial state where it was always present. Next, one has to implement in LUMLOG a "down-graded" version of the LL matrix element, which has a complete $\mathcal{O}\left(\gamma^{2}\right)$ and incomplete $\mathcal{O}\left(\gamma^{3}\right)$ - incomplete in exactly the same way as in the multi-photon BHLUMI 4.x. Let us call it for short "truncated matrix element". It is not completely trivial to obtain the truncated matrix element, because it requires integration of the BHLUMI matrix element over transverse photon momenta, keeping all terms up to $\mathcal{O}\left(\alpha^{3}\right)$ in the LL approximation. This integration was actually done in Refs. [5,10] and we use the resulting truncated matrix element in the present LUMLOG. In the following we shall give explicitly the complete truncated matrix element as implemented in the present LUMLOG.

Let us start with the new master formula for the small-angle Bhabha total crosssection, where in addition to the initial state, we have also the final state bremsstrahlung in the $\mathcal{O}\left(\alpha^{r}\right)$ LL collinear approximation:

$$
\begin{aligned}
\sigma_{\mathrm{LL}}^{(r)}= & \int_{z_{\min }}^{1} d z_{1} \int_{z_{\min }}^{1} d z_{2} \int_{0}^{1} d z_{3} \int_{0}^{1} d z_{4} \int_{0}^{1} d \xi^{*} \int_{0}^{2 \pi} d \phi D^{(r)}\left(\frac{\gamma}{2}, z_{1}\right) D^{(r)}\left(\frac{\gamma}{2}, z_{2}\right) \\
& D^{(r)}\left(\frac{\gamma}{2}, z_{3}\right) D^{(r)}\left(\frac{\gamma}{2}, z_{4}\right) \frac{d \sigma^{\text {Born }}}{d \phi d \xi^{*}}\left(\phi, \xi^{*}\right) \Theta_{\xi_{a}}^{\xi_{b}}\left(\xi_{1}\right) \Theta_{\xi_{a}}^{\xi_{b}}\left(\xi_{2}\right) .
\end{aligned}
$$


Figure 1: Leading logarithmic kinematics.

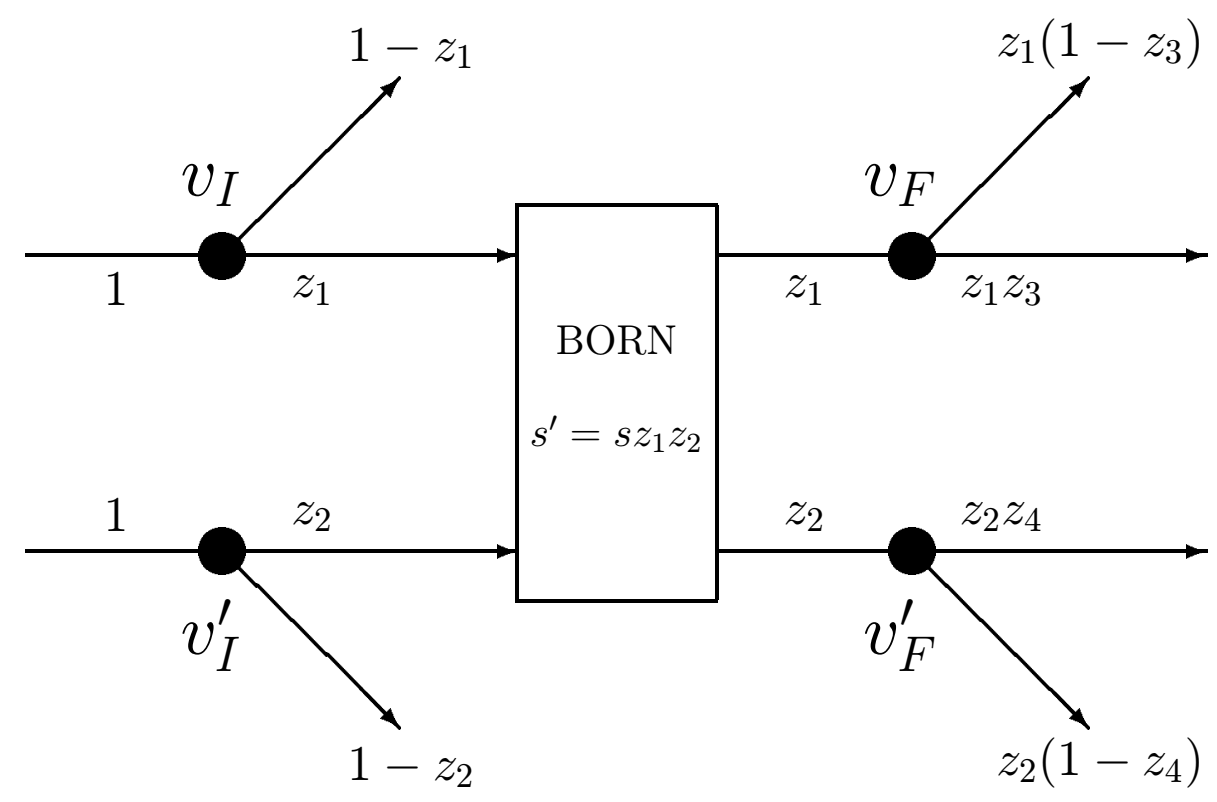

Here $\phi$ is the azimuthal angle around the beams, $z_{1}, z_{2}$ denote fractions of the energy carried by the beams after emission of the initial state photons, $z_{3}, z_{4}$ denote fractions of the energy carried by $e^{ \pm}$in the process of the emission of the final state photons, (see fig. 1 for illustration) and the Born cross-section with pure $t$-channel photon exchange reads

$$
\frac{d \sigma^{\text {Born }}}{d \phi d \xi^{*}}(\xi, \phi) \equiv \frac{2 \alpha^{2}}{s z_{1} z_{2}} \frac{1+\left(1-\xi^{*}\right)^{2}}{2\left(\xi^{*}\right)^{2}}
$$

where $\vartheta^{*}$ in $\xi^{*}=\left(1-\cos \vartheta^{*}\right) / 2=|t| /\left(s z_{1} z_{2}\right)$ is defined as the scattering angle in the LL hard scattering rest frame. The built-in event selection is defined by $\Theta_{\xi_{a}}^{\xi_{b}}(\xi)=\Theta(\xi-$ $\left.\xi_{a}\right) \Theta\left(\xi_{b}-\xi\right)$ in terms of variable $\xi=(1-\cos \vartheta) / 2$ where $\vartheta$ is defined in the laboratory system. All notation is essentially the same as in Ref. [18]. The non-singlet LL structure functions $D^{(r)}(\gamma, z)$ up to $r=3$, with exponentiation, are from Ref. [21] and are also given explicitly in Ref. [18].

The extension of the Monte Carlo algorithm to the case with the final state bremsstrahlung is straightforward. Variables $z_{3}, z_{4}$ can be generated independently of $z_{1}, z_{2}$ because event selection does not depend on $z_{3}$ and $z_{4}$. The case without exponentiation is more complicated than the case with exponentiation. We implement in the new LUMLOG $\mathcal{O}\left(\gamma^{2}\right)$ structure functions without exponentiation, using a MC algorithm very similar to that in Ref. [18], i.e. using an auxiliary small parameter $\varepsilon$ in order to emulate the deltalike parts of the distributions. All of the above is a straightforward extension of what is described in Ref. [18], so we do not discuss it in more detail.

The cross section corresponding to BHLUMI with the matrix element of $\mathcal{O}\left(\alpha^{2}\right)$ in- 
tegrated analytically over the transverse photon momenta, keeping terms ${ }^{3}$ up to $\mathcal{O}\left(\gamma^{3}\right)$, reads as follows:

$$
\begin{aligned}
\sigma_{\mathrm{LL}}^{\mathrm{BHL}}= & \int_{z_{\min }}^{1} d z_{1} \int_{z_{\min }}^{1} d z_{2} \int_{0}^{1} d z_{3} \int_{0}^{1} d z_{4} \int_{0}^{1} d \xi^{*} \int_{0}^{2 \pi} d \phi \frac{d \sigma^{\mathrm{Born}}}{d \phi d \xi^{*}}\left(\phi, \xi^{*}\right) \Theta_{\xi_{a}}^{\xi_{b}}\left(\xi_{1}\right) \Theta_{\xi_{a}}^{\xi_{b}}\left(\xi_{2}\right) \\
& z_{3} z_{4} f_{0}\left(\gamma / 2, v_{I}\right) f_{0}\left(\gamma / 2, v_{F}\right) f_{0}\left(\gamma / 2, v_{I}^{\prime}\right) f_{0}\left(\gamma / 2, v_{F}^{\prime}\right) e^{Y\left(\gamma, v_{F}\right)+Y\left(\gamma, v_{F}^{\prime}\right)} \\
& \left\{B\left[\bar{\beta}_{0}\right](\gamma)+\sum_{K=I, F} \frac{B\left[\bar{\beta}_{1 K}^{(2)}\right]\left(\gamma, v_{K}\right)}{f_{0}\left(\gamma / 2, v_{K}\right)}+\sum_{L=I, F} \frac{B\left[\bar{\beta}_{1 L}^{(2)}\right]\left(\gamma, v_{L}^{\prime}\right)}{f_{0}\left(\gamma / 2, v_{L}^{\prime}\right)}\right. \\
& +\sum_{K, L=I, F} \frac{B\left[\bar{\beta}_{2 U L}^{(2)}\right]\left(\gamma, K, L, v_{K}, v_{L}^{\prime}\right)}{f_{0}\left(\gamma / 2, v_{K}\right) f_{0}\left(\gamma / 2, v_{L}^{\prime}\right)} \\
& \left.+\sum_{K, L=I, F} \frac{B\left[\bar{\beta}_{2 U U}^{(2)}\right]\left(\gamma, K, L, v_{K}, v_{L}\right)}{f_{0}\left(\gamma / 2, v_{K}\right) f_{0}\left(\gamma / 2, v_{L}\right)}+\sum_{K, L=I, F} \frac{B\left[\bar{\beta}_{2 L L}^{(2)}\right]\left(\gamma, K, L, v_{K}^{\prime}, v_{L}^{\prime}\right)}{f_{0}\left(\gamma / 2, v_{K}^{\prime}\right) f_{0}\left(\gamma / 2, v_{L}^{\prime}\right)}\right\} .
\end{aligned}
$$

The following notation is used

$$
\begin{aligned}
& v_{I}=\left(1-z_{1}\right) z_{3}, \quad v_{F}=1-z_{3}, \quad v_{I}^{\prime}=\left(1-z_{2}\right) z_{4}, \quad v_{F}^{\prime}=1-z_{4}, \\
& Y(\gamma, v) \equiv-\gamma \ln (1-v)+\frac{1}{4} \gamma, \quad F(\gamma) \equiv \frac{\exp (-C \gamma)}{\Gamma(1+\gamma)}, \quad f_{0}(\gamma, v) \equiv F(\gamma) \gamma v^{\gamma-1}, \\
& B\left[\bar{\beta}_{0}\right](\gamma)=1+\gamma+\frac{1}{2} \gamma^{2}, \\
& B\left[\bar{\beta}_{1 K}^{(2)}\right](\gamma, v)=\bar{R}_{K}^{(2)}(\gamma, v), \quad K=I, F \\
& B\left[\bar{\beta}_{2 U U}^{(2)}\right]\left(\gamma, I, I, v_{I}, v_{F}\right)=f_{0}\left(\gamma / 2, v_{F}\right) \frac{1}{8} \gamma^{2} v_{I}^{\gamma / 2}\left(d_{0}^{*}\left(v_{I}\right)+\frac{1}{2} \gamma d_{1}^{*}\left(v_{I}\right)\right) \\
& B\left[\bar{\beta}_{2 U U}^{(2)}\right]\left(\gamma, F, F, v_{I}, v_{F}\right)=f_{0}\left(\gamma / 2, v_{I}\right) \frac{1}{8} \gamma^{2} v_{F}^{\gamma / 2}\left(d_{0}^{*}\left(v_{F}\right)+\frac{1}{2} \gamma d_{1}^{*}\left(v_{F}\right)\right) \\
& B\left[\bar{\beta}_{2 U U}^{(2)}\right]\left(\gamma, I, F, v_{I}, v_{F}\right)=\frac{1}{8} \gamma^{2} v_{I}^{\gamma / 2} v_{F}^{\gamma / 2}\left[k_{I F}^{*}\left(v_{I}, v_{F}\right)+\frac{1}{2} \gamma w_{I F}^{*}\left(v_{I}, v_{F}\right)\right] \\
& B\left[\bar{\beta}_{2 U L}^{(2)}\right]\left(\gamma, K, L, v_{K}, v_{L}^{\prime}\right)=\bar{R}_{K}^{(1)}\left(\gamma, v_{K}\right) \bar{R}_{L}^{(1)}\left(\gamma, v_{L}^{\prime}\right), \quad K, L=I, F
\end{aligned}
$$

\footnotetext{
${ }^{3}$ Next-to-leading terms are set to zero in analytical expressions after analytical integration over the transverse photon momenta.
} 
where

$$
\begin{aligned}
& \bar{R}_{I}^{(2)}(\gamma, u)=F\left(\frac{1}{2} \gamma\right) u^{\frac{1}{2} \gamma}\{\gamma(-1 / 2+(1 / 4) u) \\
& \quad+\gamma^{2}(-1 / 2+(1 / 8) u)+\gamma^{2} \ln (1-u)(1 / 8-(1 / 16) u-1 /(8 u)) \\
& \quad+\gamma^{3}(-(3 / 32) u)+\gamma^{3} \ln (1-u)(-1 / 32+(1 / 32) u) \\
& \left.\quad+\gamma^{3} \ln (1-u)^{2}(1 / 32-(1 / 64) u-1 /(32 u))+\gamma^{3} L i_{2}(u)(1 / 16-(1 / 32) u)\right\} \\
& \bar{R}_{F}^{(2)}(\gamma, u)=F\left(\frac{1}{2} \gamma\right) u^{\frac{1}{2} \gamma}\{\gamma(-1 / 2+(1 / 4) u) \\
& \quad+\gamma^{2}(-1 / 2+(1 / 8) u)+\gamma^{2} \ln (1-u)(-1 / 8+(1 / 16) u+1 /(8 u)) \\
& \quad+\gamma^{3}(-(1 / 32) u)+\gamma^{3} \ln (1-u)(+1 / 32-(1 / 32) u) \\
& \left.\quad+\gamma^{3} \ln (1-u)^{2}(-1 / 32+(1 / 64) u+1 /(32 u))+\gamma^{3} L i_{2}(u)(-1 / 16+(1 / 32) u)\right\} \\
& \bar{R}_{I}^{(1)}(\gamma, u)=\bar{R}_{F}^{(1)}(\gamma, u)=F\left(\frac{1}{2} \gamma\right) u^{\frac{1}{2} \gamma}\left\{\gamma(-1 / 2+(1 / 4) u)+\gamma^{2}(-(1 / 8) u)+\gamma^{3}(1 / 16) u\right\}
\end{aligned}
$$

and

$$
\begin{aligned}
w_{I F}^{*}\left(v_{1}, v_{2}\right) & =-\frac{1}{2} \frac{v_{1}}{1-v_{2}}+\ln \left(1-v_{1}\right)\left[-\frac{1}{2} \frac{1}{1-v_{2}}+\frac{1}{4} \frac{v_{1}}{1-v_{2}}+\frac{1}{4} \frac{v_{1}}{\left(1-v_{2}\right)^{2}}\right], \\
k_{I F}^{*}\left(v_{1}, v_{2}\right) & =k_{F I}^{*}\left(v_{2}, v_{1}\right)=\frac{1}{2}-\frac{1}{2} \frac{1}{1-v_{2}}+\frac{1}{4} \frac{v_{1}}{1-v_{2}}+\frac{1}{4} \frac{v_{1}}{\left(1-v_{2}\right)^{2}}, \\
d_{0}^{*}(v) & =\frac{1}{2} v+\frac{1}{2}\left(1-\frac{v}{2}\right) \ln (1-v), \\
d_{1}^{*}(v) & =-\frac{3}{4} v-\frac{1}{4}(1-v) \ln (1-v)+\left(-\frac{1}{2}+\frac{1}{4} v\right) L i_{2}\left(\frac{-v}{1+v}\right) .
\end{aligned}
$$

Here, $C$ is Euler's constant. The $B\left[\bar{\beta}_{2 L L}^{(1)}\right]$ is functionally identical to $B\left[\bar{\beta}_{2 U U}^{(1)}\right]$.

It is worth while to underline that the differential cross section in eq. (3) does not factorize into a product of the four functions, each dependent on one of $z_{i}=1,2,3,4$, as is the case for eq. (1). It is generally expected, because the integrand in eq. (3) misses some $\mathcal{O}\left(\gamma^{3}\right)$ terms $^{4}$. The difference between the cross sections of eq. (1) and of eq. (3) represents the missing $\mathcal{O}\left(\gamma^{3}\right)$ in BHLUMI. Since LUMLOG is an event generator, we can therefore estimate its size for arbitrary event selection. It turns out to be numerically negligible, below $2 \times 10^{-4}$, see Ref. [5], for a typical LEP luminometer, so we conclude that adding the missing $\mathcal{O}\left(\gamma^{3}\right)$ to the BHLUMI matrix element is not a high priority.

\section{Description of input and output data}

In the following we give a detailed and complete account of the input data. Wherever it was possible, the input is backward compatible with the version 2.x of Ref. [2], so the

\footnotetext{
${ }^{4}$ The version of eq. (3) truncated to $\mathcal{O}\left(\gamma^{2}\right)$ does factorize, of course.
} 
experienced user of BHLUMI will find it easy to understand.

\subsection{Input in initialization mode}

\begin{tabular}{|c|c|}
\hline Parameter & Meaning \\
\hline Npar (1)=KeyOpt & $\begin{array}{l}1000 * \text { KeyGen }+100 * \text { KeyRem }+10 * \text { KeyWgt }+ \text { KeyRnd, } \\
\text { general option switch, where KeyGen }=3 \text { for this (BHLUM4) sub- } \\
\text { generator. KeyRnd }=1,2 \text { implies use of RANMAR or RANECU } \\
\text { random number generator. For KeyRem }=0,1 \text { photon removal is } \\
\text { ON, OFF. They are equivalent but KeyRem }=0 \text { is obligatory in the } \\
\text { case of } Z \text { contribution switched on. If KeyWgt }=0 \text { then constant } \\
\text { total weight WtMod=1 events are produced (standard option for } \\
\text { detector studies), for KeyWgt } 1 \text { variable-weight (weighted) events } \\
\text { are provided (useful option for all kinds of tests) and for KeyWgt }=2 \\
\text { additional variable-weight events are also provided below mini- } \\
\text { mum transfer (useful for test of completeness of the phase space). } \\
\text { In the cases KeyWgt }=1,2 \text { the user may also exploit weights other } \\
\text { than the principal weight WtMod, see common block } / \text { WgtAll/ }\end{array}$ \\
\hline $\operatorname{Npar}(2)=$ KeyRad & $\begin{array}{l}=1000 * \text { KeyZet }+100 * \text { KeyUpd }+10 * \text { KeyMod }+ \text { KeyPia, } \\
\text { option switch determining the type of QED matrix element used } \\
\text { to calculate the principal total weight WtMod. For KeyZet }=0,1 \text {, } \\
\text { the } Z \text { boson contribution is OFF, ON. Out of KeyUpd }=0,1,2 \text { only } \\
\text { KeyUpd }=0 \text { is for standard usage. The other KeyUpd }=1,2 \text { are for spe- } \\
\text { cial tests where photon emission is allowed from the upper } / \text { lower } \\
\text { line only. KeyMod }=1,2 \text { determines type of QED matrix element, } \\
\text { KeyMod }=1 \text { is a version completely backward compatible with BH- } \\
\text { LUMI } 2.0 x, \text { KeyMod }=2 \text { is the version } 4 . x \text {, which is now DEFAULT! } \\
\text { KeyPia }=0,1,2,3 \text { specifies the type of photon vacuum polariza- } \\
\text { tion and } s \text {-channel photon. For KeyPia }=0 \text { these contributions are } \\
\text { OFF, it is used only in semi-analytical tests. For KeyPia }=1,2,3 \\
\text { vac. pol. is implemented according to Ref. [ } 7] \text { (as in BHLUMI } \\
2.01) \text {, Ref. [8] and Ref. [22]. Results for KeyPIA=2,3 are practi- } \\
\text { cally equivalent. }\end{array}$ \\
\hline $\operatorname{Xpar}(1)=$ CMSene & $\sqrt{s}$, centre-of-mass $(\mathrm{CM})$ energy in $\mathrm{GeV}$ \\
\hline $\operatorname{xpar}(2)=\operatorname{trmin}$ & Minimum $t$-channel transfer $\left|t_{\text {min }}\right|$ in $\mathrm{GeV}^{2}$ \\
\hline $\operatorname{Xpar}(3)=\operatorname{trmax}$ & Maximum $t$-channel transfer $\left|t_{\max }\right|$ in $\mathrm{GeV}^{2}$ \\
\hline $\operatorname{Xpar}(4)=e p s C M$ & $\begin{array}{l}\varepsilon \text {, dimensionless infrared cut on } \mathrm{CM} \text { energy of soft real photons, } \\
E_{\text {phot }}>\varepsilon \sqrt{s} / 2 \text {; for angles above } 20 \mathrm{mrad} \text {, the recommended range } \\
\text { is } 10^{-7}<\varepsilon<10^{-4} \text {. }\end{array}$ \\
\hline
\end{tabular}

Table 1: List of input parameters of BHLUM4 sub-generator.

The input data are transferred to BHLUMI through Xpar and Npar parameters in the initialization mode, for MODE=-1. The meaning of the entries $\operatorname{Npar}(1)$ and $\mathrm{Xpar}(1)$ is almost the same for all three sub-generators, but the other entries have a quite different 


\begin{tabular}{|c|c|}
\hline Parameter & Meaning \\
\hline Npar (1)=KeyOpt & $\begin{array}{l}=1000 * \text { KeyGen }+10 * \text { KeyWgt }+ \text { KeyRnd, } \\
\text { general option switch, where KeyGen }=2 \text { for this (LUMLOG) sub- } \\
\text { generator, KeyRnd }=1,2 \text { implies use of RANMAR or RANECU } \\
\text { random number generator. Both KeyWgt }=1,2 \text { options provide } \\
\text { variable-weight (weighted) events. For KeyWgt }=2 \text { weighted events } \\
\text { down to zero angle (below TminL) are generated }- \text { with this option } \\
\text { one may cover the complete phase space. }\end{array}$ \\
\hline Npar (2) =KeyRad & $\begin{array}{l}=100 * \text { KeyFin }+10 * \text { KeyTes }+ \text { KeyBlo, } \\
\text { option switch determining the type of QED matrix element used to } \\
\text { calculate the principal total weight WtMod. The user may exploit } \\
\text { weights other than WtMod, see common block } / \text { WgtAll/, described } \\
\text { in table } 3 \text {. For KeyFin }=0,1 \text {, the final state collinear radiation is } \\
\text { OFF, ON. Normally KeyTes }=0 \text { while KeyTes } 1 \text { is for tests only - } \\
\text { the QED electron structure functions replaced with (unrealistic) } \\
\text { testing functions }(1-z)^{1 / 2} \text {. The KeyBlo switch controls the defi- } \\
\text { nition of the big logarithm } L \text { in the calculation: for recommended } \\
\text { choice KeyBlo=3 we use } L=\ln \left(s^{\prime} \xi^{\star} / m_{e}^{2}\right)-1 \text { and for KeyBlo=4 we } \\
\text { have } L=\ln \left(s \xi_{a} / m_{e}^{2}\right)(\operatorname{cruder} \text { but legitimate choice). }\end{array}$ \\
\hline $\mathrm{Xpar}(1)=$ CMSene & $\sqrt{s}$, centre-of-mass $(\mathrm{CM})$ energy in $\mathrm{GeV}$ \\
\hline $\mathrm{Xpar}(2)=\mathrm{TminL}$ & Minimum $\vartheta$ electron/positron scattering angle in units of degree \\
\hline $\operatorname{Xpar}(3)=\operatorname{TmaxL}$ & Maximum $\vartheta$ electron/positron scattering angle in units of degree \\
\hline $\mathrm{Xpar}(4)=\mathrm{xk} 0$ & $\begin{array}{l}k_{0} \text {, dimensionless infrared cut on the CM system energy of soft } \\
\text { real photons, } E_{\text {phot }}>k_{0} \sqrt{s} / 2 \text { for not-exponentiated versions of } \\
\text { the calculation. Recommended range is } 10^{-7}<k_{0}<10^{-4} \text {. }\end{array}$ \\
\hline $\operatorname{Xpar}(5)=x k m a x$ & $\begin{array}{l}k_{\max } \text { determines the minimum effective mass } s^{\prime} \text { of the final state } \\
\text { electron and positron: } s^{\prime}>s\left(1-k_{\max }\right) \text {. Note that } k_{\max }=1 \text { is } \\
\text { allowed but we recommend } k_{\max } \leq 0.9999 \text {. }\end{array}$ \\
\hline
\end{tabular}

Table 2: List of input parameters of LUMLOG sub-generator

meaning. We therefore describe the exact meaning of Xpar and Npar in tables 1 and 2 , separately for the BHLUM4 and LUMLOG sub-generators. The input of the OLDBIS subgenerator is exactly the same as in versions 2.x, and the corresponding table can be found in Ref. [2]. The choice of sub-generator is made according to the KeyGen=1, 2, 3 switch, see description of Npar (1).

Other input parameters, such as the electron mass $m_{e}=0.0005111 \mathrm{GeV}$, QED coupling constant $\alpha=1 / 137.03604$, etc., are defined inside the program, in subroutine filbh2, or locally in the PARAMETER statements.

\subsection{Output in event generation mode}

In the event generation mode, for $\mathrm{MODE}=0$, both Xpar and Npar are ignored and the single Monte Carlo event accompanied by weights is provided to the user in 


\begin{tabular}{|c|c|c|}
\hline \multicolumn{3}{|c|}{ BHLUM4 } \\
\hline Entry & Type of cross section & Emission line \\
\hline WtSet (1) & $\begin{array}{l}\text { Principal weight =WtMod; it depends on KeyPia } \\
\text { KeyZet }\end{array}$ & upper + lower \\
\hline WtSet (11) & $Z$ contribution without vacuum polarization & upper + lower \\
\hline WtSet (12) & $Z$ contribution with vacuum polarization & upper + lower \\
\hline $\operatorname{WtSet}(30,31,32)$ & $\mathcal{O}\left(\alpha^{0}\right)_{\exp }, \mathcal{O}\left(\alpha^{1}\right)_{\exp }$ and $\mathcal{O}\left(\alpha_{\sim}^{2}\right)_{\exp }$ total & upper line only \\
\hline WtSet $(35,36)$ & $\mathcal{O}\left(\alpha^{1}\right) \exp$ version of $\tilde{\beta}_{0}$ and $\tilde{\beta}_{1}$ & upper line only \\
\hline WtSet $(37,38,39)$ & $\mathcal{O}\left(\alpha^{2}\right) \exp$ version of $\tilde{\beta}_{0}, \tilde{\beta}_{1}$ and $\tilde{\beta}_{2}$ & upper line only \\
\hline WtSet $(40,41,42)$ & $\mathcal{O}\left(\alpha^{0}\right)_{\exp }, \mathcal{O}\left(\alpha^{1}\right)_{\exp }$ and $\mathcal{O}\left(\alpha^{2}\right)_{\exp }$ total & upper + lower \\
\hline WtSet $(43,44)$ & $\mathcal{O}\left(\alpha^{1}\right) \exp$ version of $\tilde{\beta}_{0}$ and $\tilde{\beta}_{1}$ & upper + lower \\
\hline WtSet $(45,46)$ & $\mathcal{O}\left(\alpha^{1}\right)_{\exp } \tilde{\beta}_{1}$ upper and lower contribution & upper + lower \\
\hline WtSet $(47,48,49)$ & $\mathcal{O}\left(\alpha^{2}\right)_{\exp }$ version of $\tilde{\beta}_{0}, \tilde{\beta}_{1}$ and $\tilde{\beta}_{2}$ & upper + lower \\
\hline WtSet $(50,51)$ & $\mathcal{O}\left(\alpha^{2}\right)_{\exp } \tilde{\beta}_{1}$ upper and lower contributions & upper + lower \\
\hline WtSet $(52,53,54)$ & $\begin{array}{l}\mathcal{O}\left(\alpha^{2}\right)_{\exp } \tilde{\beta}_{2} \text { upper } \times \text { lower, upper and lower } \\
\text { contributions }\end{array}$ & upper + lower \\
\hline WtSet $(60,61,62)$ & $\mathcal{O}\left(\alpha^{0}\right), \mathcal{O}\left(\alpha^{1}\right)$ and $\mathcal{O}\left(\alpha^{2}\right)$ total & upper line only \\
\hline WtSet $(70,71,72)$ & $\mathcal{O}\left(\alpha^{0}\right), \mathcal{O}\left(\alpha^{1}\right)$ and $\mathcal{O}\left(\alpha^{2}\right)$ total & upper + lower \\
\hline
\end{tabular}

Table 3: Explanation of parallel weights in WtSet list for BHLUM4 sub-generator. The listed weights correspond to matrix element type $(A)$. The entries for type $(B)$ matrix element have the index shifted by 100 . See ref. [3] for the definitions of the matrix element. For example WtSet (41) corresponds to the $\mathcal{O}\left(\alpha^{1}\right)_{\text {exp }}$ exponentiated matrix element type $(\mathrm{A})$ and $W t \operatorname{Set}(141)$ corresponds to $\mathcal{O}\left(\alpha^{1}\right)_{\exp }$ type (B). The principal total weight WtMod=WtSet (1) corresponds to weight WtSet (142). For nonzero KeyPia and/or KeyZet the weight WtMod=WtSet(1) is multiplied by a factor corresponding to photon vacuum polarization and the $Z$ contribution is added. The other weights are not affected.

COMMON / MomSet / p1(4), q1(4),p2(4), q2(4), phot $(100,4)$, nphot COMMON / WgtAll / WtMod,WtCru1,WtCru2, WtSet(300)

Users of the previous version of BHLUMI should remember that the size of the matrix WtSet is now increased from 100 to 300 !

As in the previous version, matrices $\mathrm{p} 1$ and $\mathrm{p} 2$ represent four-momenta of incoming (beam) and outgoing $e^{+}$while $q 1$ and $q 2$ represent four-momenta of incoming (beam) and outgoing $e^{-}$. List of $n=$ nphot photon four-momenta is encoded in phot.

For standard applications (detector simulation) the user will work with constantweight events, obtained by setting KeyWgt=0. In this case the principal weight of each event is set to WtMod=1D0. Note that in this case the entire /WgtAll/ should be essentially ignored. (In fact, in order to prevent the user from using /WgtAll/ by mistake, we set its content to zero in this case.)

The user may sometimes wish to run our program with variable-weights, KeyWgt = 1,2 - this also speeds up the calculation. Here we envisage two levels of sophistication in the use of the program. For unsophisticated use of the program one takes events with the total weight WtMod and one should remember that the type of QED matrix element 


\begin{tabular}{|l|l|l|}
\hline \hline \multicolumn{3}{|c|}{ LUMLOG } \\
\hline Entry & Type of cross section & r.c.c. \\
\hline WtSet $(1,2,3,4)$ & $\begin{array}{l}\mathcal{O}\left(\alpha^{0}\right)_{\exp }, \mathcal{O}\left(\alpha^{1}\right)_{\exp }, \mathcal{O}\left(\alpha^{2}\right)_{\exp } \text { and } \mathcal{O}\left(\alpha^{3}\right)_{\exp } \\
\text { total }\end{array}$ & No \\
WtSet $(5)$ & $\mathcal{O}\left(\alpha^{3}\right)_{\exp }$ total & Yes \\
WtSet $(11,12,13)$ & $\mathcal{O}\left(\alpha^{0}\right), \mathcal{O}\left(\alpha^{1}\right)$ and $\mathcal{O}\left(\alpha^{2}\right)$ total \\
\hline \multicolumn{2}{|c|}{ LL emulation of BHLUMI in LUMLOG } \\
\hline WtSet $(40,41,42)$ & $\mathcal{O}\left(\alpha^{0}\right)_{\exp }, \mathcal{O}\left(\alpha^{1}\right)_{\exp }$ and $\mathcal{O}\left(\alpha^{2}\right)_{\exp }$ total & No \\
WtSet $(43,44)$ & $\mathcal{O}\left(\alpha^{1}\right)_{\exp }, \tilde{\beta}_{0}, \tilde{\beta}_{1}$ & No \\
WtSet $(45,46)$ & $\mathcal{O}\left(\alpha^{1}\right)_{\exp }, \tilde{\beta}_{1}$ upper/lower line components & No \\
WtSet $(47,48,49)$ & $\mathcal{O}\left(\alpha^{2}\right)_{\exp }, \tilde{\beta}_{0}, \tilde{\beta}_{1}$ and $\tilde{\beta}_{2}$ & No \\
WtSet $(50,51)$ & $\mathcal{O}\left(\alpha^{2}\right)_{\exp }, \tilde{\beta}_{1}$, upper/lower line components & No \\
WtSet $(52,53,54)$ & $\mathcal{O}\left(\alpha^{2}\right)_{\exp }, \tilde{\beta}_{2}$, upper $\times$ lower, upper and lower line & No \\
& $\operatorname{components}$ & \\
\hline \hline
\end{tabular}

Table 4: Explanation of parallel weights in WtSet list for LUMLOG sub-generator. The principal total weight WtMod is related to WtSet (4). The use of the running coupling constant (r.c.c.) is indicated.

in WtMod is determined by KeyRad=Npar $(2)^{5}$. The advanced user of the program may, in the same Monte Carlo run, use a variety of alternative weights defined as WtAlt $(\mathrm{J})=$ WtCru $1 * W t C r u 2 * W t \operatorname{Set}(\mathrm{J})$, where each $\mathrm{J}$ corresponds to one of many versions of the QED matrix element, in various perturbative orders and with various contributions switched on/off. This is explained in more detail, separately for each type of sub-generator, in table 3 for the BHLUM4 sub-generator and in table 4 for the LUMLOG sub-generator. Let us note that for the BHLUM4 sub-generator (run in the variable-weight mode) the matrix element without exponentiation (see weights WtSet $(60-61,70-71)$ ) does not include the $Z$-contribution and vacuum polarization - the user himself/herself has to combine the relevant weights (see subroutines model2 and m2agzi). The user may also consult the examples of the user programs demo.f and demo2.f described in the next section.

It is important to know that for variable-weight events the final state four-momenta may be ill-defined in events with $^{6}$ WtMod=0 or WtCru1*WtCru2 $=0$. In order to avoid an unnecessary crash of the user program the kinematical calculations should be protected by the appropriate IF (WtMod.NE.ODO) THEN ... ENDIF conditional statement. This will also speed up the user histogramming program a little bit.

Finally, let us also note that the backward compatibility in the matrix element of BHLUM4 with version 2.01 of Ref. [2] is kept, i.e. the matrix element of the version 2.01 can be accessed optionally by means of setting KeyMod $=1$, see table 1 , and in this case table 4 of Ref. [2] applies for the description of the output weights WtMod and WtAlt (J), see also examples described in the next section.

\footnotetext{
${ }^{5}$ N.B.: For the constant-weight events, exactly this weight is used for the rejection inside the MC generator.

${ }^{6}$ In the present version all four-momenta are set to zero for such events.
} 


\section{Program topography, usage and samples of results}

Figure 2: Topography of the distribution directory.

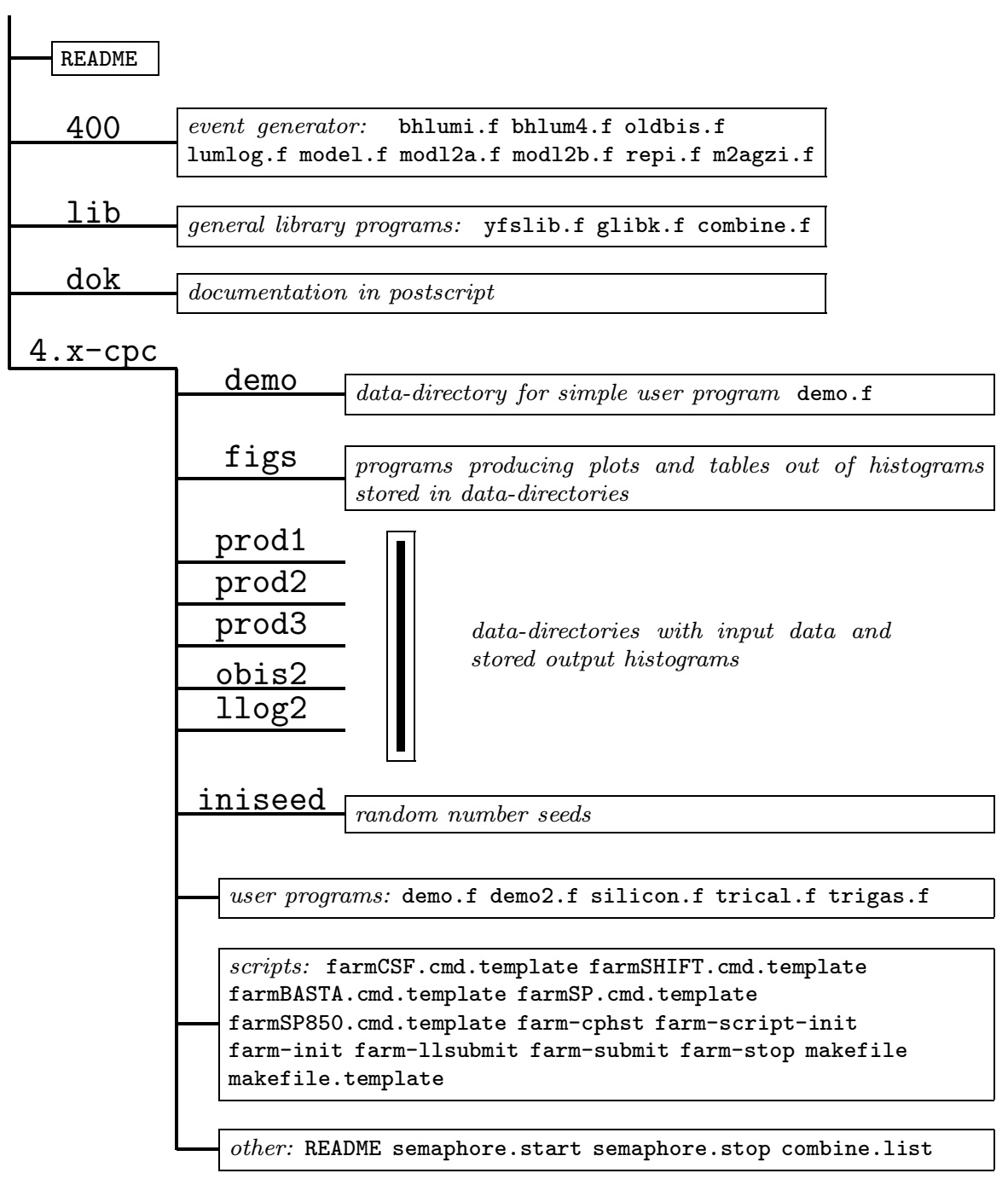

The main objective of the BHLUMI MC event generator is to calculate very precisely the total cross section of the small-angle Bhabha process for arbitrary event selection (ES). The distribution package of the present BHLUMI version 4.04 was prepared in such a way that its content should help the user to accomplish this task. Having the above in mind we included in the distribution package not only the BHLUMI MC event generator itself (which consists of three sub-generators BHLUM4, LUMLOG and OLDBIS), but also two examples of the user programs and several examples of the output results. We envisage that the user will run these demonstration user programs and use them as a starting point to develop his/her own user program(s). The output data (tables, plots and printouts) should help the user in the non-trivial task of checking whether the BHLUMI MC event 
generator, as implemented on a given platform, is really reproducing these outputs with a precision of a fraction of per mille! In the following we shall describe the "topography" of the distribution package and how to run the demonstration user programs. We shall not enter too much into descriptions of the subprograms of the BHLUMI MC event generator because it is quite similar to that in Ref. [2] for the earlier version 2.02.

The entire distribution package of the present BHLUMI 4.04 is organized into a single UNIX directory with three sub-directories 400, $1 i b, 4 . x-c p c$ containing programs and the sub-directory dok containing documentation (postscripts of the relevant papers), see Fig. 2.

\subsection{Event generator and simple user program}

The complete Monte Carlo event generator BHLUMI (consisting of the three standard sub-generators BHLUM4, OLDBIS and LUMLOG) is located in 400 and lib. The subdirectory 400 contains the essential parts of the MC generator, which generate fourmomenta, and a collection of the matrix elements. The sub-directory lib contains programming tools, including the histogramming package glibk. This part of the package (400 and $\mathrm{lib}$ ) is self-contained, each sub-directory has its own makefile capable to compile its content. The user may develop his/her own main program and link it with the object files from the 400 and lib. A standard sequence of calls such as

PROGRAM main

IMPLICIT DOUBLE PRECISION $(\mathrm{a}-\mathrm{h}, \mathrm{o}-\mathrm{z})$

* Histograms in labeled common

COMMON / cglib / b(50000)

* Common blocks with output events from bhlumi MC event generator COMMON / momset / p1(4), q1(4),p2(4), q2(4), phot $(100,4)$, nphot

COMMON / wgtall / wtmod, wtcru1, wtcru2, wtset(300)

* Input parameters

DIMENSION xpar(100), npar(100)

* Initialization of histogramming package --

CALL glimit(50000)

(book histograms)

CALL bhlumi (-1, xpar, npar)

DO IEVENT $=1,10000$

CALL bhlumi ( 0,xpar,npar)

(fill histograms)

ENDDO

CALL bhlumi ( 2, xpar,npar)

(print histograms)

END

will produce a series of MC events and the user will be able to calculate the total cross section and arbitrary distributions for any ES. The input data encoded in XPAR and 
NPAR were described in the previous section. However, instead of building up his/her own user program from scratch, following the above example, we recommend to follow the entry-level example of the user program provided by authors of BHLUMI and described in the following.

Figure 3: This plot is in 4.x-cpc/demo/demo.tex. The distribution d(sigma)/d(theta) [nanobars/radians].

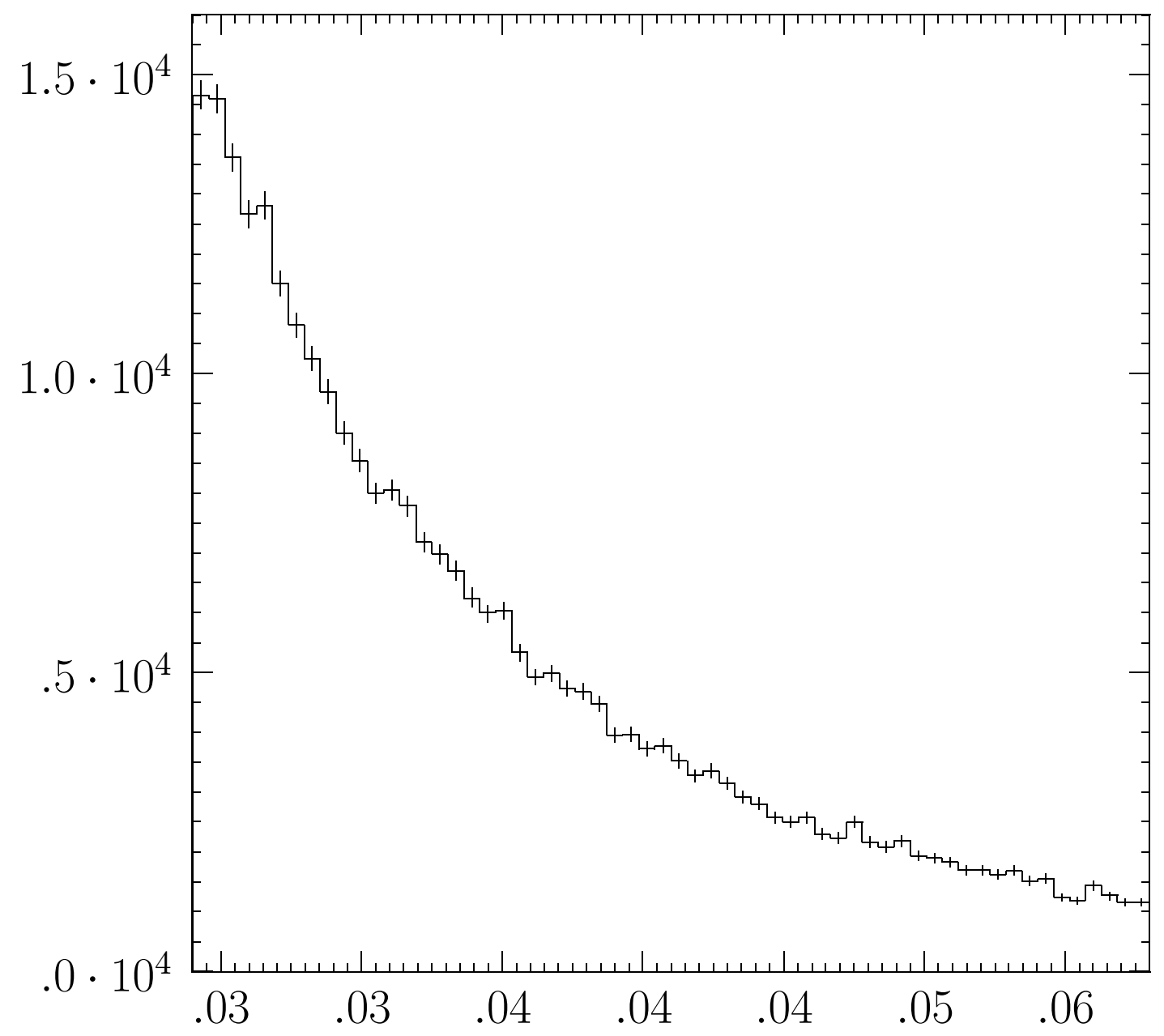

It should be stressed that the sub-directory $4 . \mathrm{x}$-cpc should never be treated as a part of the event generator. In the sub-directory $4 . \mathrm{x}$-cpc we implemented a rich collection of examples of running BHLUMI. The event generator part, 400 and lib, is totally independent of them.

There are two sophistication levels in the examples of the user programs. We recommend very strongly every user to treat the entry-level user program demo.f in $4 . x$-cpc as 
an instructive and complete example of running BHLUMI. The other program demo2.f in $4 . x-c p c$ should be used as an example of a more sophisticated use of the BHLUMI event generator. We shall describe both in this section.

The execution of the entry-level user program demo.f can be initiated by the following set of simple commands:

$\mathrm{cd} 4 . \mathrm{x}-\mathrm{cpc}$

make demo

The user program demo.f has the following interesting features:

- It includes several examples of input data for the multi-photon BHLUM4 subgenerator and shows how to run BHLUMI with constant weights and variable weights.

- It shows how to select MC events and calculate the total cross section for two examples of event selections BARE1 and CALO2 defined in Ref. [11]. At the end of the output demo/demo. output the user finds the cross sections corresponding to the respective entry in Table 14, for event selection BARE1, $z_{\min }=0.5$, and in Table 18, for event selection CALO2, $z_{\min }=0.5$, in Ref. [11], see also the very end of the sample output of Appendix A.

- It shows how to calculate properly normalized (in nanobarns) single-dimensional distributions. They are stored in demo/demo.tex and one of them ${ }^{7}$, the angular distribution of the electron, is shown in Fig. 3, for a small statistics of $10^{4}$ events. For the run with variable weights there are two more output distributions, see demo.f and demo/demo.tex; one of them is the distribution of the principal weight wtmod and the other one is produced using the technique of the alternative weights described in Section 3.2.

The demo directory contains sample outputs from the MC sample of $10^{4}$ events, which can be used for the initial check of whether BHLUMI runs properly on a given platform. In order to do meaningful comparisons with the tables of Ref. [11], more events have to be generated. The sample outputs included in demo are obtained on the IBM Power Series machine under AIX 4.

\subsection{Advanced user program and numerical benchmarks}

The user of the program may, as described above, keep only the program $4 . x-c p c /$ demo.f and the sub-directory $4 . \mathrm{x}-\mathrm{cpc} /$ demo, forget the rest of $4 . \mathrm{x}-\mathrm{cpc}$ and develop his/her own main user program using $4 . \mathrm{x}-\mathrm{cpc} /$ demo.f as a starting point. The other example of the user program $4 . \mathrm{x}-\mathrm{cpc} /$ demo2.f and directories other than $400,1 \mathrm{ib}, 4 . \mathrm{x}-\mathrm{cpc} / \mathrm{demo}$ are ignored in such a case. What is the role of the demo2.f and related sub-directories and why do we include them in the distribution package? We are going to address this question in the following.

\footnotetext{
${ }^{7}$ The other one, not shown here, is the normalized distribution of the photon energy.
} 
Table 5: This is from the output file 4.x-cpc/figs/BeMaTabs.tex. Total cross sections for various symmetric Wide-Wide, event selections from BHLUMI, in nanobarns. BHLUM4 $4_{p h t}$ and $B H L U M 4_{Z V P}$ denote standard multi-photon BHLUMI event generator. OBI+LMG denotes so-called OLDBIS+LUMLOG h.o. additive recipe while BHLPV follows SABSPV multiplicative recipe realized using OLDBIS and LUMLOG cross section. Only in $\mathrm{BHLUM} 4_{Z V P}$ are $Z$ exchange, up-down interference and vacuum polarization switched on. Centre-of-mass energy is $\sqrt{s}=92.3 \mathrm{GeV}$. Not calculated cross sections set to zero.

\begin{tabular}{||c|c|c|c|c||}
\hline \hline$z_{\min }$ & BHLUM $_{p h t}$ & OBI+LMG & BHLPV & BHLUM $_{Z V P}$ \\
\hline \multicolumn{5}{|c||}{ (a) BARE1, Born $=175.977 \mathrm{nb}$} \\
\hline .100 & $166.882 \pm .006$ & $166.674 \pm .014$ & $166.738 \pm .016$ & $.000 \pm .000$ \\
.300 & $165.365 \pm .006$ & $165.189 \pm .014$ & $165.251 \pm .016$ & $.000 \pm .000$ \\
.500 & $162.522 \pm .006$ & $162.366 \pm .014$ & $162.436 \pm .016$ & $.000 \pm .000$ \\
.700 & $155.660 \pm .006$ & $155.518 \pm .014$ & $155.606 \pm .015$ & $.000 \pm .000$ \\
.900 & $137.334 \pm .006$ & $137.203 \pm .013$ & $137.325 \pm .013$ & $.000 \pm .000$ \\
\hline \multicolumn{5}{|c|}{ (c) CALO2, Born $=140.018 \mathrm{nb}$} \\
\hline .100 & $131.826 \pm .006$ & $131.641 \pm .013$ & $131.718 \pm .014$ & $136.963 \pm .006$ \\
.300 & $131.441 \pm .006$ & $131.283 \pm .013$ & $131.375 \pm .014$ & $136.564 \pm .006$ \\
.500 & $130.719 \pm .006$ & $130.593 \pm .013$ & $130.714 \pm .014$ & $135.815 \pm .006$ \\
.700 & $127.960 \pm .006$ & $127.807 \pm .013$ & $128.019 \pm .014$ & $132.950 \pm .006$ \\
.900 & $118.785 \pm .006$ & $118.203 \pm .012$ & $118.856 \pm .013$ & $123.407 \pm .006$ \\
\hline \multicolumn{5}{|c|}{ (d) SICAL2, Born $=140.018 \mathrm{nb}$} \\
\hline .100 & $132.807 \pm .006$ & $132.593 \pm .013$ & $132.624 \pm .014$ & $137.984 \pm .006$ \\
.300 & $132.545 \pm .006$ & $132.416 \pm .013$ & $132.453 \pm .014$ & $137.711 \pm .006$ \\
.500 & $131.976 \pm .006$ & $131.976 \pm .013$ & $132.028 \pm .014$ & $137.121 \pm .006$ \\
.700 & $128.663 \pm .006$ & $128.617 \pm .013$ & $128.778 \pm .014$ & $133.680 \pm .006$ \\
.900 & $119.006 \pm .006$ & $118.491 \pm .012$ & $119.111 \pm .013$ & $123.637 \pm .006$ \\
\hline \multicolumn{5}{|c|}{ (e) SICAL, Born=140.018 nb } \\
\hline .100 & $132.216 \pm .006$ & $132.085 \pm .013$ & $132.137 \pm .014$ & $137.370 \pm .006$ \\
.300 & $132.181 \pm .006$ & $132.053 \pm .013$ & $132.107 \pm .014$ & $137.334 \pm .006$ \\
.500 & $131.920 \pm .006$ & $131.822 \pm .013$ & $131.886 \pm .014$ & $137.062 \pm .006$ \\
.700 & $129.228 \pm .006$ & $129.084 \pm .013$ & $129.223 \pm .014$ & $134.267 \pm .006$ \\
.900 & $120.341 \pm .006$ & $119.845 \pm .012$ & $120.327 \pm .013$ & $125.025 \pm .006$ \\
\hline \hline \multicolumn{5}{|c}{}
\end{tabular}

The role of $4 . \mathrm{x}-\mathrm{cpc} /$ demo2. $\mathrm{f}$ and related sub-directories is to demonstrate how certain important numerical results of Refs. $[3,5,11]$ were obtained in practice. Ultimately, our aim is to allow the user to reproduce exactly (up to a statistical MC error) all important numerical results from BHLUMI published up to now. The user programs that were employed to obtain these important numerical results in Refs. $[3,5,11]$ and other papers are incorporated in the user program 4.x-cpc/demo2.f. The sub-directories prod1, prod2, prod3, $\log 2$, obis 2 of the $4 . x-c p c$ directory contain the relevant input data and receive all output results from the MC runs - they are data directories. The data directories prod1, prod2, prod3 are for the BHLUM4 multi-photon sub-generator, $1 \log 2$ for LUMLOG and obis2 for the OLDBIS sub-generator. The other sub-directory $4 . \mathrm{x}$-cpc/figs 
contains programs that produce selected important plots and tables of Refs. $[3,5,11]$. The user is, in this way, capable to reproduce by himself/herself many important published tables and plots obtained using the present BHLUMI MC event generator. However, to reproduce all these results from scratch requires several thousands of hours of CPU on a fast workstation. In order to be able to run immediately the graphics programs in $4 . \mathrm{x}-\mathrm{cpc} / \mathrm{figs}$ we have stored in the data directories a collection of the archive-histograms files from our own long runs. (See the latter part of this section on how to run these graphics programs.) On the other hand, in order to help the advanced user, who intends to reproduce all the results, we have included in $4 . x$-cpc several shell-scripts and makefiles which will help to run $4 . x-c p c / d e m o 2 . f$ in parallel on any typical workstation-farm such as SP-2 and CSF at CERN. Finally, let us also note that in $4 . x-c p c$ we included programs for several event selections defined/used in Refs. $[3,5,11,17,18]$, in particular for all four event selections BARE1, CALO1, CALO2 and SICAL2 of Refs. [11] and for the SICAL and OPSIW event selections of Refs. [3,9]. They are encoded in the programs silicon.f, trigas.f and trical.f in the directory $4 . x-c p c$. Let us now describe in more detail how to run the user program $4 . x-c p c / d e m o 2 . f$.

The execution of the entry-level user program demo2. $f$ for the multi-photon event subgenerator BHLUM4 can be initiated for the input data set $4 . \mathrm{x}-\mathrm{cpc} / \mathrm{prod} 2 / \mathrm{prod} 2$. data by the following set of simple commands:

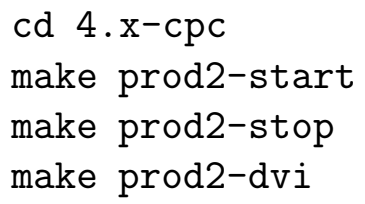

The second command, make prod2-start, causes the program to be compiled and then the main program starts to generate MC events using the multi-photon event sub-generator BHLUM4 in the background process. The execution may continue up to a maximum number of events defined in $4 . \mathrm{x}-\mathrm{cpc} / \mathrm{prod} 2 / \operatorname{prod} 2$. data or, optionally, it can be stopped interactively by issuing the third command, make prod2-stop. At the end of the run all histograms are stored in the file $4 . \mathrm{x}-\mathrm{cpc} / \mathrm{prod} 2 / \mathrm{bhl}$.hst. In fact, histograms are written into this file every $10^{5}$ events anyway. The last command make prod2-dvi initiates compilation and execution of the program $4 . x$-cpc/figs/prod2.f, which reads histograms from $4 . \mathrm{x}-\mathrm{cpc} / \mathrm{prod} 2 / \mathrm{bhl}$. hst and shows on the screen plots of various distributions. In fact it produces the $\mathrm{AT}_{\mathrm{E} X}$ file $4 . \mathrm{x}-\mathrm{cpc} / \mathrm{figs} / \mathrm{prod} 2$. tex which is then processed and viewed on the screen using $x d v i$. The storage file $4 . x-c p c / p r o d 2 / b h 1 . h s t$ and another file, $4 . \mathrm{x}-\mathrm{cpc} / \mathrm{prod} 2 /$ semaphore, contains in addition to user-defined histograms, the complete information on the status of the internal parameters of the event generator. Thanks to this arrangement the MC run can be restarted and continued, without any loss of accumulated statistics, and stopped at any suitable moment, with the following simple commands:

make prod2-cont

make prod2-stop 
Figure 4: This is from the output file 4.x-cpc/figs/BeMaFigs.tex. The difference $d_{3}=$ (BHLPV BHLUMI.4x)/Born for the SICAL event selection as a function of the energy cut $1-U_{\min }$. BHLPV is an emulation of SABSPV using OLDBIS and LUMLOG. The dashed box marks 1.0 per mille precision limits.

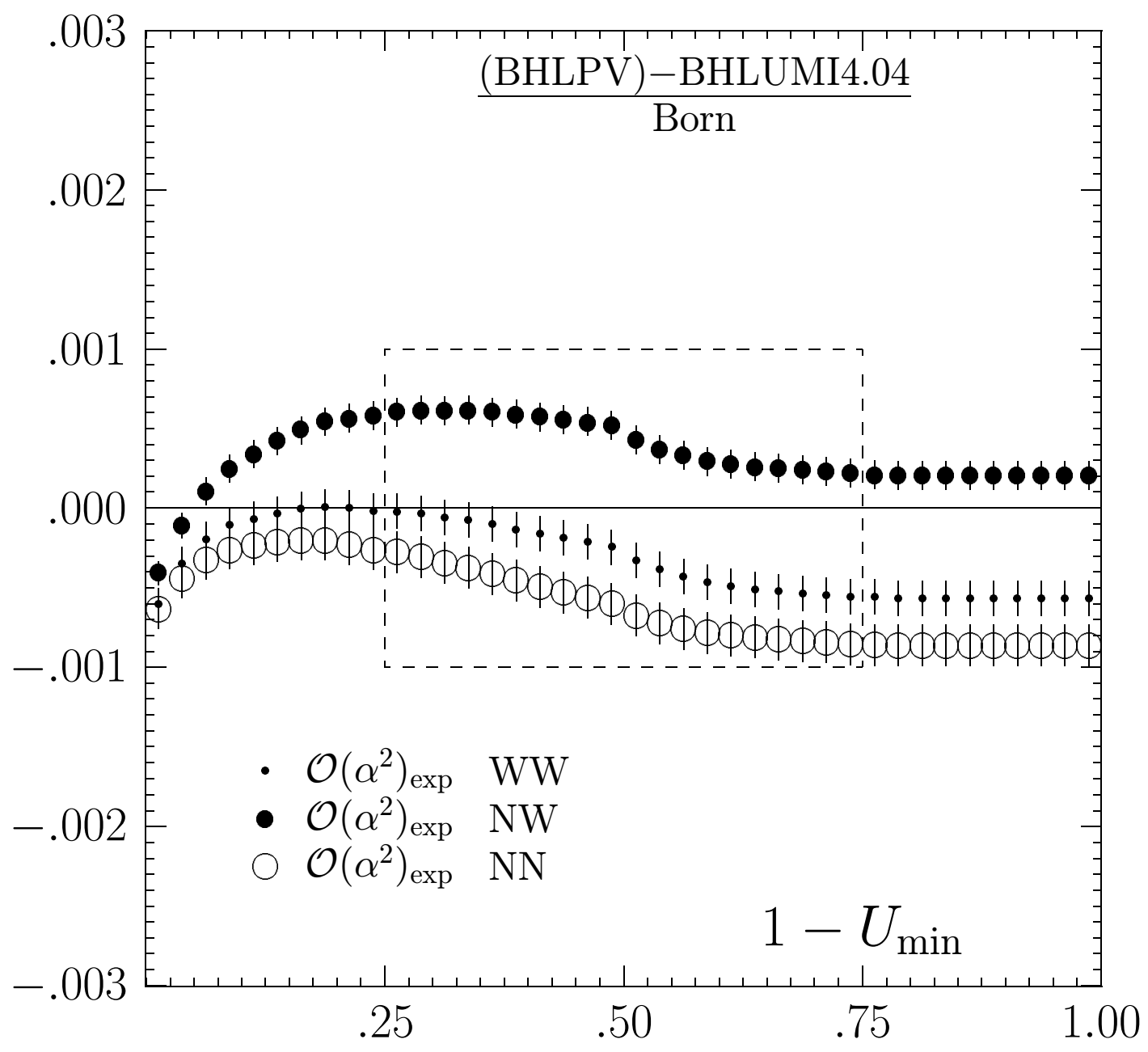

The user will most probably want to make contact with the published values from the BHLUMI 4.03(4) program. Since, typically, these results involve comparisons of BHLUMI with OLDBIS and LUMLOG, it is necessary to run the other two sub-generators and store histograms in the data sub-directories obis 2 and $1 \log 2$ by executing the following simple commands:

$$
\begin{aligned}
& \text { cd } 4 . x-c p c \\
& \text { make } 1 \text { log2-start } \\
& \text { make obis2-start }
\end{aligned}
$$

Partial results may be pre-viewed as before with: 
Figure 5: This is from the output file 4.x-cpc/figs/BeMaFigs.tex. The difference $d_{3}=$ (OLDBIS - LUMLOG L.o. $_{\text {. }}-$ BHLUMI.4x)/Born for the SICAL event selection as a function of the energy cut $1-U_{\min }$. The dashed box marks 1.5 per mille precision limits.

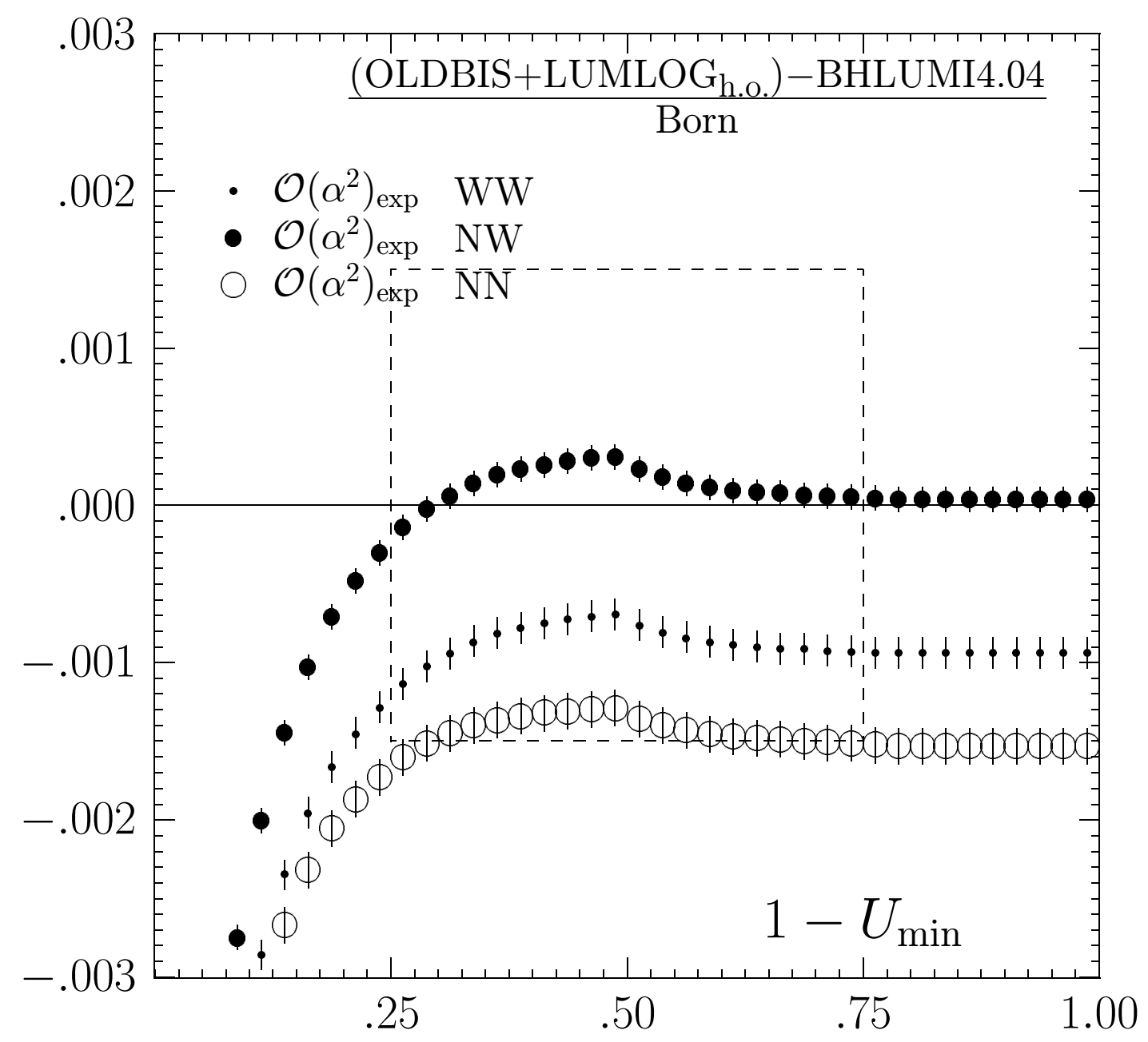

make $1 \log 2-d v i$

make obis2-dvi

Finally, after running for long enough, the whole table of cross sections shown in Table 5 can be obtained, and viewed on the screen, by executing:

cd $4 . x-c p c / f i g s$

make BeMaTabs-dvi

The results of Table 5 are for about $2 \times 10^{9}$ events for each of the three sub-generators. The main purpose of the above set of runs, which results in Table 5, is to help the user to 
get convinced that the program is running correctly and that he/she is able to reproduce the known published cross-sections from BHLUMI. In short, Table 5 constitutes a very important numerical benchmark of the actual BHLUMI program. The table addresses the important problem of the precision of BHLUMI only partly. We shall come back to this question later in this section. What are the results listed in Table 5? As we see, they are calculated for the symmetric, so-called wide-wide version of the four types of event selection, BARE1, CALO2, SICAL2, which are defined/used in Ref. [11] and SICAL in Ref. [3]. Let us recall that BARE1 is an unrealistic non-calorimetric ES introduced in order to compare with some theoretical semi-analytical calculations, for example those of Refs. [23] and [24]. The other two, CALO2 and SICAL2, are calorimetric semi-realistic ES's which are closer to the true experimental ES's, and SICAL is a very close representation of the real SICAL luminometer of the ALEPH Collaboration and to the actual selection criteria used in their data analysis.

In Table 5 BHLUMI results are shown in two versions: first, for the pure photonic case, i.e. without the $Z$ boson and vacuum polarization (VP) contributions and the second with the $Z$ boson and VP included. Furthermore, in Table 5 we include the results from LUMLOG and OLDBIS, more precisely the additive combination OLDBIS $+\mathrm{LUMLOG}_{\text {h.o. }}$ defined in Ref. [18], and the multiplicative combination BHLPV, which is an exact emulation of the SABSPV program of Ref. [13] (see also a description of SABSPV in Ref. [11]). The additive OLDBIS + LUMLOG ${ }_{\text {h.o. }}$ is, according to Refs. [11,12], practically identical with the results from the BHAGEN95 program of Ref. [25], see also Refs. [11,26,27], while the multiplicative prescription BHLPV should be equal exactly to the results of the SABSPV MC, see Refs. [13,16,28]. The entries in Table 5 agree within a statistical error of $\left(\sim 2 \times 10^{-4}\right)$ with the entries in Tables 14 and 18 of Ref. [11]. The absolute values of the cross sections for the SICAL ES were not given in Ref. [3], so our Table 5 supplements this paper and constitutes an important numerical benchmark of the BHLUMI 4.04 program. Last but not least, let us remind the reader that contrary to BHLUMI, which is a full-scale event generator, the OLDBIS + LUMLOG $G_{h . o}$ and BHLPV (SABSPV) calculations are not event generators but only $\mathrm{MC}$ calculations (albeit for arbitrary ES).

Both additive and multiplicative combinations of LUMLOG and OLDBIS have been used extensively to cross-check how precise the total cross section from the BHLUMI program is, and we therefore come gradually to this very basic question. The recent improved precision estimate of BHLUMI 4.03(4) of $0.11 \%$ is based mainly on the comparison of the BHLUMI and SABSPV results, see Refs. [11,12], for symmetric and asymmetric event selections. In the BHLUMI package, in addition to the figs/BeMaTabs.f program that produces Table 5, we have included another graphics program figs/BeMaFigs.f, which can be executed as follows

cd 4.x-cpc/figs

make BeMaFigs-dvi

and produces Fig. 4 stored in figs/BeMaFigs.tex. Note that this new program uses the same three output histogram files prod2/bhl.hst, obis $2 /$ bhl.hst and 1 log $2 /$ bhl.hst 
as the previous program BeMaTabs.f. In the new plot, we show the difference between BHLPV and multi-photon BHLUMI for the symmetric and asymmetric type of the realistic event selection SICAL. As expected, the difference is within $0.10 \%$ for the interesting range of values of the energy cut around 0.5. As we see the SABSPV prescription is now almost fully realized using OLDBIS and LUMLOG (we do not include $Z$ and VP in our BHLPV result), with the advantage that each of the two, OLDBIS and LUMLOG, were cross-checked individually many times, with many methods, see Refs. [5,11,17,23]. Each of them thus has a well-established sub-per mille technical precision. It is interesting to see how, for the same SICAL ES, the older additive combination OLDBIS + LUMLOG $_{h . o}$ of Refs. [3,18] compares with the BHLUMI 4.04 results. Figure 5, which is also stored in figs/BeMaFigs.tex, answers this question. The difference is within $0.15 \%$, in agreement with Ref. [3].

The above results in Table 5 and Figs. 4 and 5 were obtained from long runs on the CERN SP-2 farm and reproducing them on a single workstation may take some time. We have therefore included in the data directories the archive-histogram files from these long runs. The user may therefore plot immediately the above table and plots without actually running very long $\mathrm{MC}$ runs, by invoking the following commands ${ }^{8}$ :

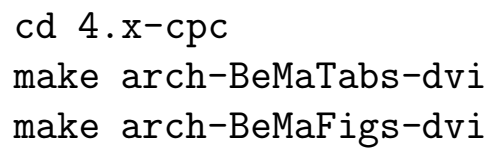

In fact, we include in $4 . \mathrm{x}-\mathrm{cpc}$ /makefile the possibility to run a graphics program, showing even more published plots from BHLUMI. In particular, we included programs producing plots on the $Z$-boson contribution of Ref. [4] and on the missing third-order photonic contributions in BHLUMI of Ref. [5]. The interested reader should consult this makefile, the README file, and the relevant programs in the directory $4 . \mathrm{x}-\mathrm{cpc} / \mathrm{figs}$.

Summarizing, in the presented distribution package the user should find all possible examples of the use of the BHLUMI event generator and should be able to reproduce many important published results.

\subsection{Running on a farm}

Finally, let us explain briefly how to run $4 . \mathrm{x}-\mathrm{cpc} /$ demo2.f on a farm of workstations in order to accumulate high statistics more efficiently. For example, in order to run for the BHLUM4 sub-generator 12 parallel jobs on the SP-2 farm at CERN, one should do the following:

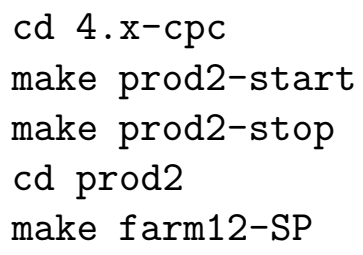

\footnotetext{
${ }^{8}$ On certain computers a double back-slash may need to be replaced by the single one. This is achieved with: cd figs; make ibm-to-hp.
} 
make 1lsubmit12

make combine12

(cd ../figs; make prod2-dvi)

The user may easily find out by inspecting $4 . \mathrm{x}-\mathrm{cpc} / \mathrm{prod} 2 /$ makefile how to query on submitted jobs, change their parameters, etc. We have included templates of scripts to run jobs on several farms. Very briefly, the command make farm12-SP creates 12 sub-directories in $4 . \mathrm{x}-\mathrm{cpc} /$ prod2 and each submitted job uses one of them in order to store histograms. The command make 1lsubmit12 submits 12 jobs, each of them with a different random number seed taken from the directory $4 . \mathrm{x}-\mathrm{cpc} /$ iniseed. The same command can be used to resubmit the programs after they terminate. As for normal runs described previously, restarting of generation is done preserving continuity of the generation. Each job will first read from the disk the histograms stored at the end of the previous job and add more events to them as if there were no break in the execution. The command make combine12 sums up the content of histograms from all sub-directories (parallel jobs) and stores the results into a single $4 . \mathrm{x}$-cpc/prod2/bhl.hst, which can be used immediately as an input to graphics programs. This command can be executed while the jobs are still running - it is thus possible to view partial results before the production jobs terminate.

\section{Conclusions}

The new version 4.04 of the program BHLUMI represents the state of the art in the calculations of the QED corrections to small-angle Bhabha and their technical and physical precision. As the previous version, it is not only a full-scale stand-alone Monte Carlo event generator, ready to use for any kind of detector study and to calculate the QED corrections to the luminosity measurement; it is also a tool-box of programs, which allows one to do all kinds of sophisticated tests and to cross-check the obtained numerical results. It is still not the final solution to the problem of getting the small-angle Bhabha cross section with the sub-per mille precision, but it represents a major step in this direction. According to Refs. $[11,12]$ its overall precision is $0.11 \%$ (down from the previous $0.16 \%$ ). The present BHLUMI package embodies this new result in the form of a MC event generator, and also includes almost all the calculational tools that were used to arrive at this new result.

\section{Acknowledgements}

Useful discussions with Drs. M. Bigi, M. Dallavalle, O. Nicrosini, A. Read, B. Pietrzyk, W. D. Schlatter, M. Skrzypek and other members of the Bhabha Working Group of the LEP2 workshop are acknowledged. We thank especially M. Bigi and A. Read for help in debugging our program. All the authors acknowledge the support and kind hospitality of the CERN Theory Division, and two of us (SJ and BFLW) are grateful to the ALEPH and OPAL Collaborations for support. 


\section{APPENDIX A}

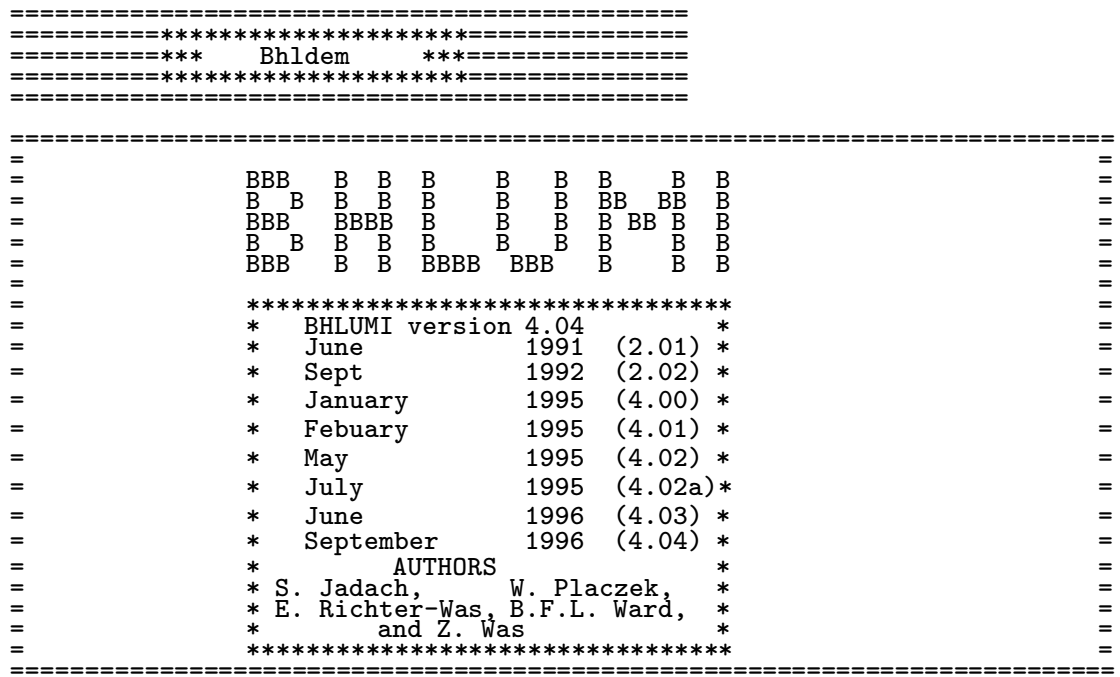

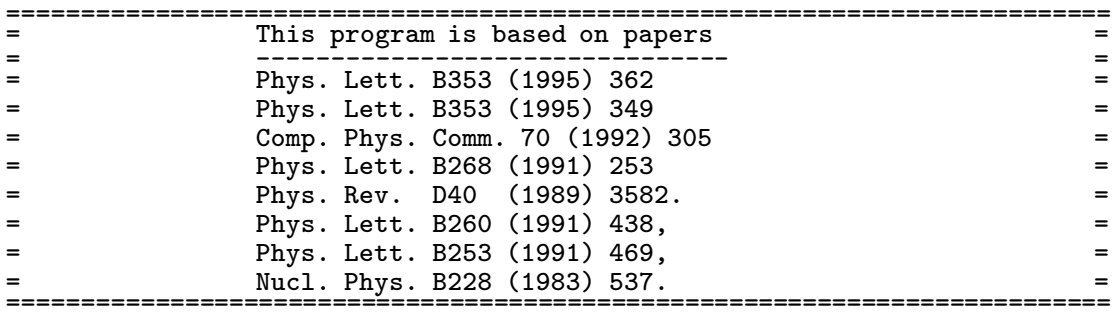

\begin{tabular}{|c|c|c|c|c|}
\hline $\begin{array}{l}= \\
= \\
=\end{array}$ & $\begin{aligned} * * * \\
\mathrm{~B} \\
* * *\end{aligned}$ & $\begin{array}{l}* * * * * * * * * * * * * * \\
\text { AMETRES } \\
* * * * * * * * * * * * * *\end{array}$ & & $\begin{array}{l}= \\
=\end{array}$ \\
\hline$=$ & 3001 & OPTIONS switch & KeyOpt & $=$ \\
\hline$=$ & 1 & rand. numb. switch & KeyRnd & $=$ \\
\hline$=$ & 0 & weighting switch & KeyWgt & $=$ \\
\hline$=$ & 0 & photon removal sw & KeyRem & $=$ \\
\hline$=$ & 1022 & RADIATION switch & KeyRad & $=$ \\
\hline$=$ & 2 & vac_pol switch & KeyPia & $=$ \\
\hline$=$ & 2 & QED mat. elm. type & KeyMod & $=$ \\
\hline$=$ & 0 & Test switch, $\operatorname{def}=0$ & KeyUpd & $=$ \\
\hline$=$ & 1 & Z contribution & KeyZet & $=$ \\
\hline$=$ & 92.30000000 & CMS energy [GeV] & CMSENE & $=$ \\
\hline$=$ & 8519.2900 & $\mathrm{CMSENE} \frown 2 \quad\left[\mathrm{GeV}^{\wedge} 2\right]$ & SVAR & $=$ \\
\hline$=$ & .60110696 & trasf_min [ [GeV^2] & TRMIN & $=$ \\
\hline$=$ & 28.626770 & trasf_max $\left[\mathrm{GeV}^{\wedge} 2\right]$ & TRMAX & $=$ \\
\hline$=$ & $.70558340 \mathrm{E}-04$ & xi_min=TRMIN/SVAR & XIMIN & $=$ \\
\hline$=$ & $.33602295 \mathrm{E}-02$ & $\mathrm{xi}$ _max $=\mathrm{TRMAX} / \mathrm{SVAR}$ & XIMAX & $=$ \\
\hline$=$ & 16.80000000 & theta_min [mrad] & THMIN & $=$ \\
\hline$=$ & 116.00000000 & theta_max [mrad] & THMAX & $=$ \\
\hline$=$ & .96256910 & theta_min & THMIN & $=$ \\
\hline$=$ & 6.64631042 & theta_max & THMAX & $=$ \\
\hline$=$ & $.10000000 \mathrm{E}-03$ & eps_CM infr. cut & EPSCM & $=$ \\
\hline$=$ & $.10000000 \mathrm{E}-05$ & delta infr. cut & DEL & $=$ \\
\hline$=$ & -.01480534 & RePi(transf_min) & REPI1 & $=$ \\
\hline $\begin{array}{l}= \\
=\end{array}$ & .00006655 & error & dREPI1 & $\begin{array}{l}= \\
=\end{array}$ \\
\hline$=$ & 00039879 & error & dREPI2 & $=$ \\
\hline$=$ & 1870 & Z-mass $\mathrm{GeV}$ & AMA & $=$ \\
\hline $\begin{array}{l}= \\
=\end{array}$ & $\begin{array}{l}2.49000000 \\
23190000\end{array}$ & Z-width GeV & GAMMZ & $\begin{array}{l}= \\
=\end{array}$ \\
\hline & & 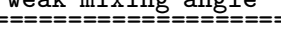 & & \\
\hline
\end{tabular}

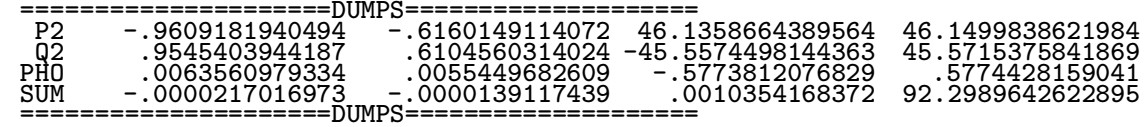



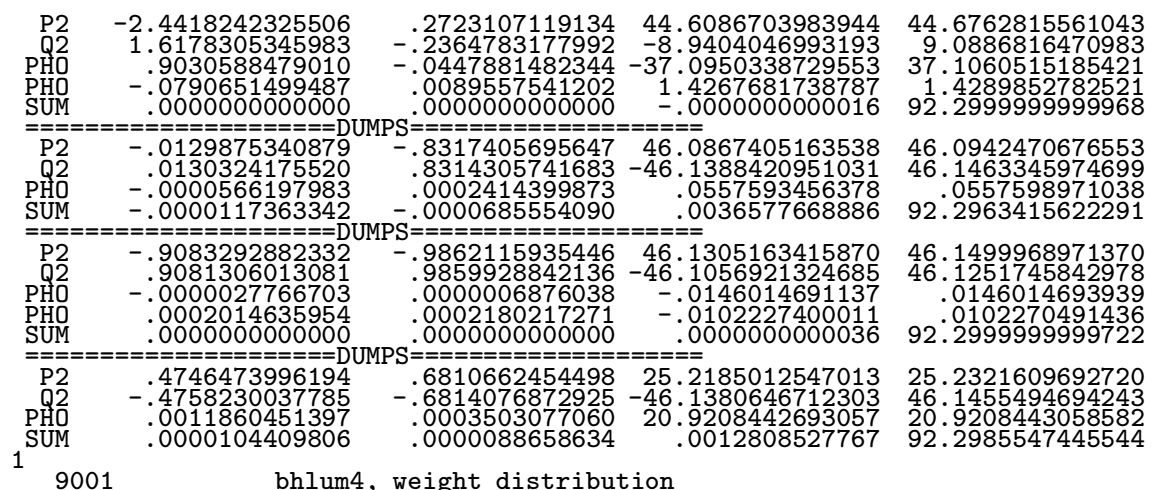

46.0942470676553
46.1463345974699

.0557598971038

46.149097130

46. 1251745842978

.0146014693939

92.0102270491436

25.2321609692720

46.1455494694243

20.9208443058582

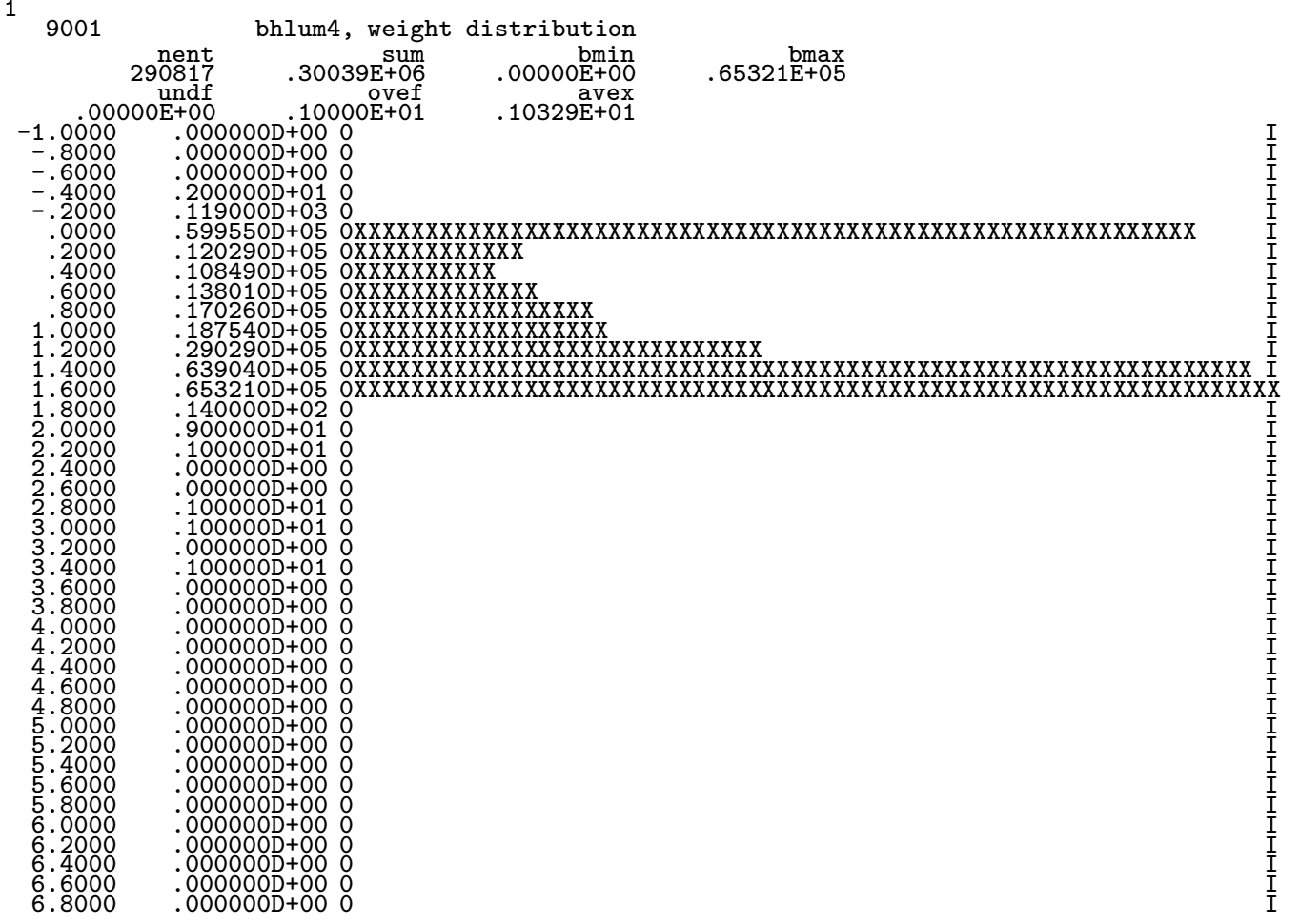

\begin{tabular}{|c|c|c|c|c|c|c|}
\hline $\begin{array}{l}= \\
= \\
=\end{array}$ & \multicolumn{4}{|c|}{ 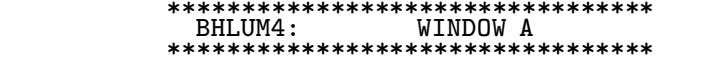 } & \multirow[b]{2}{*}{ NEVGEN } & \multirow[b]{2}{*}{ A1 } \\
\hline$=$ & 100000 & & & Accepted total & & \\
\hline$=$ & 290817 & & & Raw prior reject. & IEVENT & A2 \\
\hline $\begin{array}{l}= \\
=\end{array}$ & 438.34090 & +- & .49338970 & Xsec M.C. [nb] & $\begin{array}{l}\text { XSECMC } \\
\text { ERELMC }\end{array}$ & A3 \\
\hline$=$ & 1.03290582 & +- & .00112558 & weight M.C. & AWT & A5 \\
\hline$=$ & 121 & & & $\mathrm{WT}<0$ & NEVNEG & A6 \\
\hline $\begin{array}{l}= \\
=\end{array}$ & 3.00000000 & & & $\begin{array}{l}\text { WT>WTMAX } \\
\text { Maximum WT }\end{array}$ & $\begin{array}{l}\text { NEVUVE } \\
\text { WWMX }\end{array}$ & $\begin{array}{l}\text { A } 7= \\
\text { A8 }=\end{array}$ \\
\hline
\end{tabular}

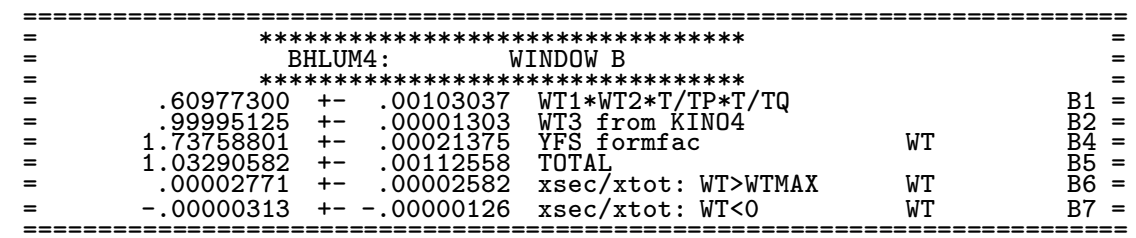

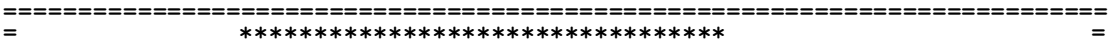




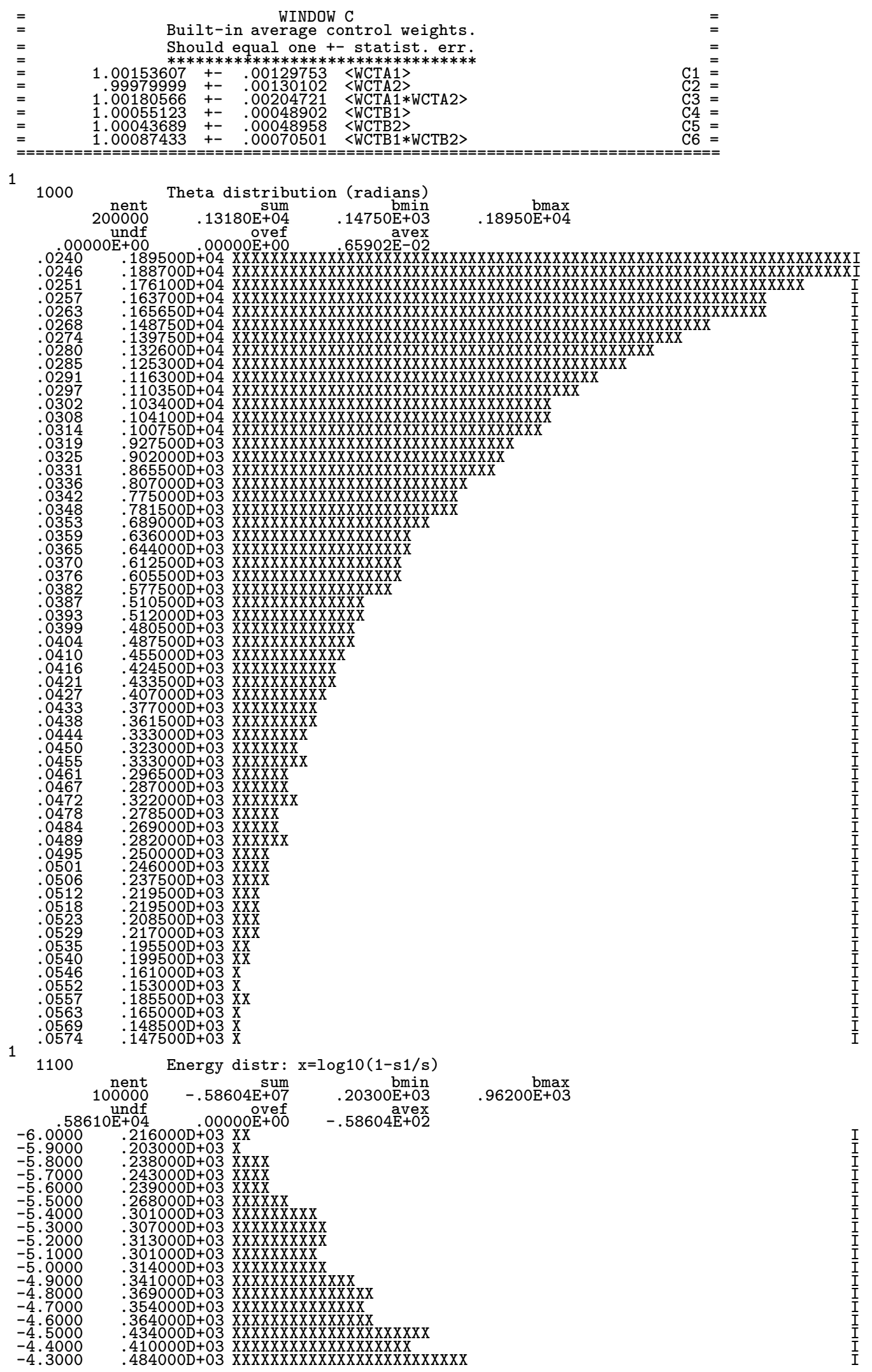




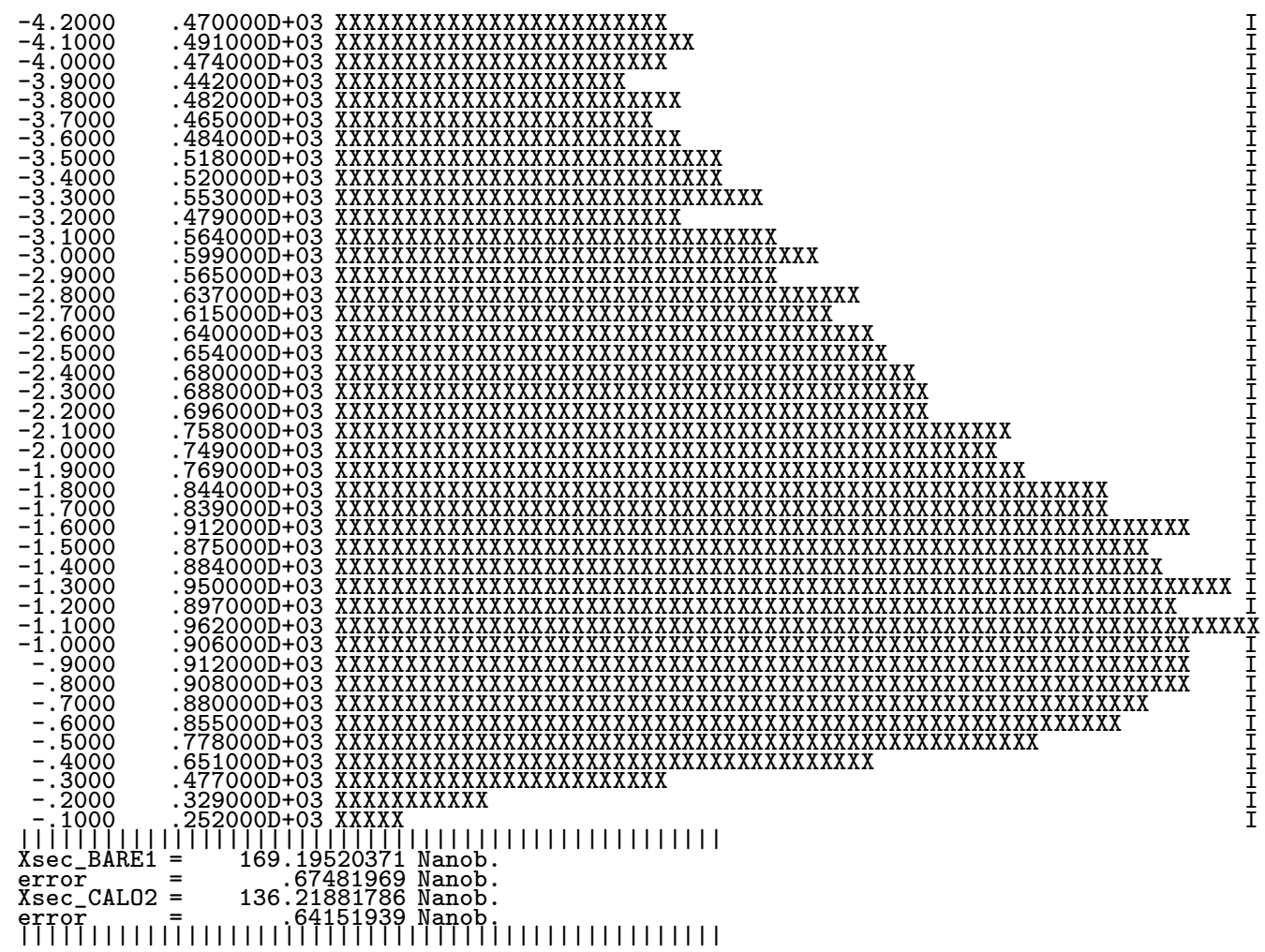

\section{References}

[1] LEP Electroweak Working Group, A Combination of Preliminary LEP Electroweak Results from the 1995 Summer Conferences, Technical report, CERN LEPEWWG/95-02, (unpublished), see also ALEPH 95-093 PHYSIC 95-086, DELPH 95-137 PHYS 562, L3 Note 1814, OPAL Technical Note TN312.

[2] S. Jadach, E. Richter-Wạs, B. F. L. Ward, and Z. Wạs, Comput. Phys. Commun. 70, 305 (1992).

[3] S. Jadach, E. Richter-Wạs, B. F. L. Ward, and Z. Wąs, Phys. Lett. B353, 362 (1995), preprint CERN-TH/95-38, see also erratum, in print.

[4] S. Jadach, W. Płaczek, and B. F. L. Ward, Phys. Lett. B353, 349 (1995), preprint CERN-TH/95-74, March 1995.

[5] S. Jadach and B. F. L. Ward, preprint CERN-TH/96-156, June 1996, Phys. Lett. B (1966) in print.

[6] H. Burkhardt and B. Pietrzyk, Phys. Lett. B356, 398 (1995).

[7] H. Burkhardt, F. Jegerlehner, G. Penso, and C. Verzegnassi, Z. Phys. C43, 497 (1989). 
[8] S. Eidelman and F. Jegerlehner, Z. Phys. C67, 585 (1995).

[9] S. Jadach et al., in Tennessee International Symposium on Radiative Corrections: Status and Outlook, Gatlinburg, Tennessee, USA, June 1994, edited by B. F. L. Ward (World Scientific, Singapore, 1995); see also CERN preprint CERN-TH.7452/94.

[10] S. Jadach and B. F. L. Ward, Semi-Analytical Third Order Calculations of the Small Angle Bhabha Cross Sections, 1996, to be published in Acta Phys. Pol.

[11] S. Jadach and O. Nicrosini (conveners of Bhabha Working Group), in Physics at LEP2, CERN Report 96-01, edited by G. Altarelli, T. Sjöstrand, and F. Zwirner (CERN, Geneva, 1996), Vol. 2, p. 229, hep-ph/9602393.

[12] A. Arbuzov et al., Phys. Lett. B383, 238 (1996), hep-ph/9605239.

[13] M. Cacciari, G. Montagna, O. Nicrosini, and F. Piccinini, Comput. Phys. Commun. 90, 301 (1995), preprint CERN-TH/95-169, hep-ph/9507245.

[14] S. Jadach, M. Melles, B. F. L. Ward, and S. A. Yost, Phys. Lett. B377, 168 (1996), hep-ph/9603248.

[15] S. Jadach, M. Melles, B. F. L. Ward, and S. Yost, in $Q C D$ and QED in Higher Order: 1996 Zeuthen Workshop on Elementary Particles, Rheinsberg, Germany, 1996, edited by J. Bluemlein (Elsevier Science Publishers, Amsterdam, 1996), preprint CERN$\mathrm{TH} / 96-176$.

[16] G. Montagna, O. Nicrosini, and F. Piccinini, Phys. Lett. B, (1996), in print; also preprint FNT/T-96/8, hep-ph/9605252.

[17] S. Jadach, E. Richter-Wạs, B. F. L. Ward, and Z. Wạs, Phys. Lett. B260, 438 (1991).

[18] S. Jadach, E. Richter-Wa̧s, B. F. L. Ward, and Z. Wạs, Phys. Lett. B268, 253 (1991).

[19] M. Caffo et al., "Bhabha Scattering", in Z Physics at LEP 1, CERN Report 89-08, edited by G. Altarelli, R. Kleiss, and C. Verzegnassi (CERN, Geneva, 1989), p. 171.

[20] S. Jadach, B. F. L. Ward, and M. Skrzypek, Phys. Lett. B257, 173 (1991).

[21] M. Skrzypek, Acta Phys. Pol. B23, 135 (1992).

[22] H. Burkhardt, in Electroweak interactions and unified theories, edited by J. Tran Than Van (Editions Frontières, Gif-sur-Yvette, 1995), in print.

[23] S. Jadach, E. Richter-Wạs, B. F. L. Ward, and Z. Wạs, Phys. Lett. B253, 469 (1991).

[24] A. Arbuzov et al., CERN preprint CERN-TH/95-313 (unpublished).

[25] M. Caffo and H. Czyż, preprint DFUB-96-20, e-Print Archive: hep-ph/9607357 (unpublished). 
[26] M. Caffo, H. Czyż, and E. Remiddi, Int. J. Mod. Phys. 4, 591 (1993).

[27] M. Caffo, H. Czyż, and E. Remiddi, Phys. Lett. B327, 369 (1994).

[28] G. Montagna, O. Nicrosini, and F. Piccinini, Comput. Phys. Commun. 78, 155 (1993), erratum, ibid. $\mathbf{7 9}$. 